\title{
ACUTE AND LONG-TERM MENTAL AND PHYSICAL SEQUELAE IN THE AFTERMATH OF TRAUMATIC EXPOSURE - SOME REMARKS ON "THE BODY KEEPS THE SCORE"
}

\author{
Hans-Peter Kapfhammer \\ Department of Psychiatry and Psychotherapeutic Medicine, Medical University of Graz, Graz, Austria
}

received: 3.7.2018;

revised: 2.8.2018;

accepted: 30.8 .2018

\section{SUMMARY}

Traumata, by definition, refer to exterior events that expose a person to experiences of overwhelming threat and catastrophe and elicit feelings of death anxiety, panic, horror, helplessness, loss of personal control, and intractability. Most affected persons respond with at least some distressing symptoms of trauma-related memory intrusions, autonomic hyperarousal, dissociation, and depression in the acute aftermath. Fortunately, the majority of traumatized individuals succeed in coping with this major stress quite well during the following weeks and months unless the process of recovery is hampered by additional adverse psychosocial circumstances, psychological disposition or biological vulnerability.

In a subgroup of persons a transition to acute and posttraumatic stress disorder or other major psychiatric disorders, e.g. depressive, anxiety, substance-related disorders may be observed. Posttraumatic stress disorders very often run a chronic course of illness enduring for many years or even life-long. The typical course of illness in PTSD is characterized not only by major psychiatric comorbidities contributing to a dramatically reduced health-related quality of life, to many deficits of psychosocial adaptation and a heightened suicide risk. It is also associated with a lot of major somatic health problems both in acute and long-term stages.

The main focus here is on this special dimension of physical comorbidities in posttraumatic disorders. Empirical evidence underscores that trauma exposure, and in particular PTSD is significantly associated with major physical health problems in addition to well-known PTSD-related psychological, behavioural, and psychosocial impairments. Both self-report-based and objective assessments emphasized significantly increased rates of somatoform/functional syndromes and physical comorbidities, premature all-cause and specific mortality rates, heightened medical utilization behaviours, major socioeconomic costs, and reduced health-related quality of life in the aftermath of trauma exposure and posttraumatic stress disorders, thus defining a major challenge to any medical care system. Complex psycho-behavioural-somatic and somato-psycho-behavioural models are needed to better understand both acute and long-term effects of a perpetuating stress system on physical health.

Key words: trauma exposure - posttraumatic stress disorder - somatic symptoms - functional bodily distress syndromes - physical comorbidity - pathophysiological pathways

\section{Introduction}

The high frequency of manifold adverse events both in modern civil and family life has sensitized public awareness in Western societies for traumatic exposures and major negative effects on health and quality of life. The ubiquitous reality of wars, genocides, natural disasters, man-made catastrophes, and attacks of terrorism has additionally sharpened this critical awareness. A forced mass migration of thousands of foreign refugees from various regions of the world is meanwhile arriving at and transgressing the borders of the European States. This globalized view on trauma is presenting an inevitable challenge to our basic self-understanding regarding human solidarity and civilization. Conceptualization of trauma in Western tradition of thinking has restricted itself predominantly to suffering states of individuals, whereas has often faded out broader psychosocial, cultural, existential and ethical contexts of trauma (Kirmayer et al. 2010). Many instances of human suffering in modern life, especially in the aftermath of trauma exposure, have increasingly come into the realm of medical competence and responsibility, in particular of psychiatry and psychotherapy. Somewhat of a surprise, the very dimension of physical morbidity as concomitant sequelae of trauma exposure has gained proper scientific recognition only in recent past.

The present paper intends to outline the physical domain of posttraumatic development in some broad lines. Starting with basic diagnostic features of posttraumatic psychological disorders and general epidemiological findings it will focus on the various somatic response patterns during acute stages of posttraumatic processing and on an ever increasing somatic comorbidity and increased premature mortality during chronic courses of posttraumatic stress disorders. And it will discuss some important pathophysiological pathways that may mediate discrete posttraumatic risks of somatoform and functional bodily distress syndromes on the one hand and of major somatic diseases on the other. The theoretical frame of reference will be a model of mind-brain-body medicine.

This article is based on a lecture at Danube Symposium "Psyche and body - A major challenge

to modern clinical psychiatry", Graz, Austria, May 26, 2018 


\section{Diagnostic, clinical and epidemiological situation at the outset}

Traumata, by definition, refer to exterior events that expose to experiences of overwhelming threat and catastrophe and elicit feelings of death anxiety, panic, horror, and helplessness. Inherent formal features of traumatic experiences are suddenly confronting, extremely threatening, unavoidable, incontrollable, and inescapable. Traumata may be either a single event that disrupts psychosocial normality by a certain moment, may appear in a cumulative way during various stages of lifespan, or may define an enduring traumatic reality without any foreseeable way out. These formal characteristics of traumatic exposures may be associated with quite different immediate and long-term outcomes. And this also applies to different types of trauma, natural disaster, man-made technical catastrophe, civil accident, terror attack, war, torture, genocide, ethnic cleansing, or sexual, physical, emotional abuse, neglect, violence in interpersonal relations, but also acute life-threatening medical conditions. All these different types of trauma may contain special codes of individual and collective understanding traumatic experiences, respectively, thus decisively determining posttraumatic cognitive, affective, behavioural and physiological processing (Kapfhammer 2017a).

Modern psychiatry has conceptualized several trauma-related diagnostic categories, acute stress disorder (ASD) and posttraumatic stress disorder (PTSD) the most prototypical variants. According to DSM-IV ASD defined a severe stress-syndrome during the first month after a traumatic event. The diagnosis was originally introduced to detect a subgroup of highly traumatized persons that probably present an increased risk of transition to PTSD later on and seemingly form a proper indication for early secondary prevention. Besides symptoms of trauma-related memory intrusion, avoidance and autonomic hyperarousal a special obligatory focus of diagnosis was on dissociative symptoms. Due to insufficient sensitivity of the former concept of ASD as proofed by empirical research DSM-5 has meanwhile altered the diagnostic algorithm in favour of a now free combination of symptoms out of various trauma-related clusters of psychopathology, however, setting a high threshold of clinical severity ( $\geq 9$ out of 14 symptoms) as prerequisite for diagnosis. DSM-5 has also significantly changed the diagnostic criteria of PTSD compared to its version in DSM-IV. The conceptual design now covers a much broader range of clinical symptom clusters. Besides traditional symptoms of intrusion and avoidance it comprises a detailed list of trauma-related negative alterations in cognitions and mood and also a more detailed cluster of autonomic hyperarousal. In addition, DSM-5 now appreciates discrete specifiers both of a dissociative subtype with prominent symptoms of depersonalization and derealisation and of a delayed onset of PTSD (Kapfhammer 2014). DSM-5 and forthcoming ICD-11 will follow a strikingly different diagnostic logic as regards trauma-related and other stress- related disorders in the future (Maercker et al. 2013). This conceptual divergence in both major diagnostic classification systems within psychiatry will have a profound impact on epidemiological key figures of trauma-related disorders (Brewin et al. 2017, Hafstad et al. 2017).

Even in ordinary contexts of modern Western societies the risk of getting exposed to various traumata at least once in a lifetime is quite high. It actually occurs often in a cumulative way. Life-time prevalence of trauma exposure is ranging from some $50 \%$ to $90 \%$ (Wittchen et al. 2009). On the other side, most persons will recover after trauma exposure within a defined period of time. Neither ASD nor PTSD can be considered fixed normative response patterns to traumatic events. Both prototypical posttraumatic disorders have to be conceptualized as results of an atypical development determined by multiple biopsychosocial factors that predispose to, start, mediate and maintain manifold psychopathological and pathophysiological pathways (Shalev 2009). Factors of both vulnerability and resilience have to be taken into account within a complex pathogenic context to elucidate the various courses both of recovery and of chronic illness (McEwen et al. 2015).

Conditional risk of PTSD after trauma exposure is quite variable. It seems to be highest in traumata shattering the core identity of a person, such as incidents of extreme violence (e.g. sexual abuse, rape, torture, military combat), but may also be very high after the message of the unexpected death of a loved person. There is a clear dose-response curve as regards severity and frequency of defined trauma exposure (sexual violence, natural disaster, military combat, terroristic attack) and conditional risk of PTSD. Men are usually exposed to more frequent traumatic events than women. Women, however, generally show a higher risk of PTSD after trauma exposure. Life-time prevalence rates of PTSD in the USA are in the range of 7-8\% (Norris \& Slone 2014). Important societal contexts and differing standards of social living have to be appreciated, when these prevalence figures are significantly lower in most West European countries on the one hand and significantly higher in most low-income countries on the other (Atwoli et al. 2015).

From an epidemiological perspective ASD and PTSD may be considered as prototypical posttraumatic disorders. After traumatic exposures, however, a significantly increased incidence of various other psychological disorders may be observed as well, among them most frequently disorders of depression, anxiety, substance use, and somatoform disorders (Reifels et al. 2017, Asselmann et al. 2018). Around one third of individuals who develop PTSD in the aftermath of trauma exposure will run a chronic course of illness often persisting for many years or even life-long (Kessler et al. 1995, Santiago et al. 2013, Morina et al. 2014, Rosellini et al. 2018). Chronic courses of PTSD demonstrate many different complications that have to be properly assessed within a biopsychosocial model. A high risk of 
psychiatric comorbidity up to some $80 \%$ in chronic courses of PTSD has to be considered, particularly depressive, anxiety, and substance use disorders (Keane et al. 2007). As a rule, these psychiatric comorbidities follow the prior manifestation of PTSD (Atwoli et al. 2015). They have to be appraised separately as regards course of illness and subsequent further complications (Steinert et al. 2015).

Major personality changes (e.g. deficits of self-care, emotional dysregulation, high-risk behaviour, grossly distorted personal responsibility, hampered self-efficacy) may result from early and later trauma exposure (Levy \& Seidel 2013). Significantly reduced healthrelated quality of life, significantly increased psychosocial disabilities (Schnurr et al. 2009), severe risks of suicidal behaviour (Pompili et al. 2013), and highly increased socioeconomic costs (Priebe et al. 2009) are further striking hallmarks of chronic PTSD.

And one other, very often neglected characteristic of chronic PTSD refers to a great many unexplained somatic symptoms, functional bodily distress syndromes, and somatoform disorders on the one side, and an excessively increased rate of comorbid physical diseases in the longrun on the other (Schnurr 2015, McLeay et al. 2017).

\section{Trauma exposure, posttraumatic stress syndromes and somatic health status}

At the outset, one basic epidemiological finding is to be stressed: From a perspective of empirical investigations there is highly significant association between trauma exposure, posttraumatic stress syndromes and physical health problems. This is equally true for studies that have been methodologically based either on patients' self-reports or objective assessments by doctors, that have conceptually referred to medical utilization behaviour, health-related quality of life, manifold syndromes of somatization or proper somatic diseases, rates of premature mortality, and that have been established for various epidemiological samples focussing on trauma exposure either in early childhood or during later adulthood. The pathway from trauma exposure to finally reduced or impaired somatic health is prevailingly mediated by a diagnostic status of PTSD. This relationship seems to be more pronounced in men than in women. Specific PTSD effects beyond those mediated by other comorbid mental disorders (e.g. depressive, anxiety, and substance use disorder) can be confirmed by analytical methods (Schnurr \& Jankowski 1999, Schnurr et al. 2007, Schnurr et al. 2014, Schnurr 2015; Pacella et al. 2013).

\section{Intricate association between trauma exposure, posttraumatic stress syndromes and somatic health status - some exemplary illustrations}

A recently published analysis of data from the Adult Psychiatric Morbidity Survey 2007 reported on some 7.403 individuals aged $\geq 16$ years out of general UK population. After adjustment for sociodemographic factors, life-time occurrence of traumatic events, rate of PTSD, and information on 20 doctor-diagnosed somatic conditions present during the past 12 months (cancer, diabetes, epilepsy, migraine, ophthalmological, otological, cardio-/cerebrovascular, pulmonary, gastrointestinal, urogenital, musculoskeletal, dermatological, infectious diseases) a strong association between PTSD and physical multimorbidity could be established (Odds ratio=2.47). The prevalence of PTSD of $2.1 \%$ in individuals with no physical condition increased to $5.4 \%$ in those with $\geq 4$ chronic physical conditions (Figure 1). Anxiety, depressive and eating disorders that had also been assessed in this representative epidemiological sample explained $35 \%, 21 \%$ and $8 \%$ of the association between PTSD and physical multimorbidity, respectively (Jacob et al. 2018).

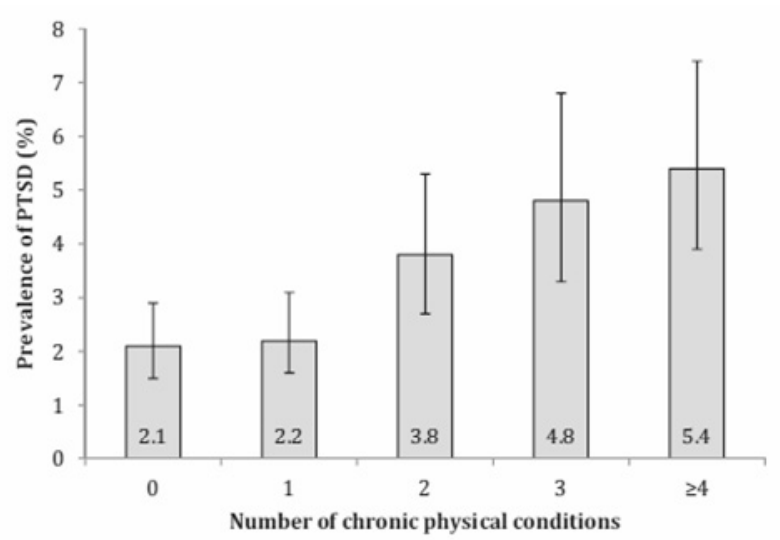

Figure 1. Association of PTSD and physical multimorbidity (Jacob et al. 2018)

Cross sectional studies are not able to differentiate between causative, confounding or mediating factors that may make up any association between traumata, posttraumatic stress disorders on the one hand, and physical conditions on the other. From a theoretical point of view several possible causal structures of the relation between PTSD and defined somatic condition (e.g. cardiometabolic disease) have to be discussed (Koenen et al. 2017; Figure 2).

Modern aetiopathogenetic understanding of both PTSD and any somatic condition or physical disease is complex and multifactorial and refutes simple linear models of causality as obsolete. This equally applies to perspectives of analysis that start either from typical exterior traumatic exposures (variant 2) (Friedman et al. 2014) or from an enduring somatic threat model of PTSD due to acute life-threatening medical events (variant 3) (Edmondson 2014). And this seems to be true as well when one takes a broad focus on general vulnerability (variant 1), considers various mediating behavioural patterns associated with PTSD (variant 4) or investigates several underpinning pathophysiological pathways that are shared both by PTSD and defined somatic condition (Koenen et al. 2017). 
1. PTSD and somatic condition share environmental / genetic factors

risk factor

2. PTSD causes somatic condition

PTSD

3. Somatic condition causes PTSD

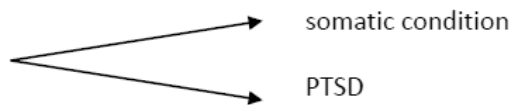

somatic condition

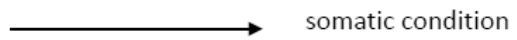

somatic condition

PTSD $\longrightarrow$ e.g. smoking $\longrightarrow$ somatic condition

5. PTSD and somatic condition interact with each other

PTSD

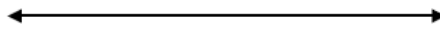

somatic condition

Figure 2. Possible causal relations in the association of PTSD and defined somatic condition (Modified: Koenen et al. 2017)

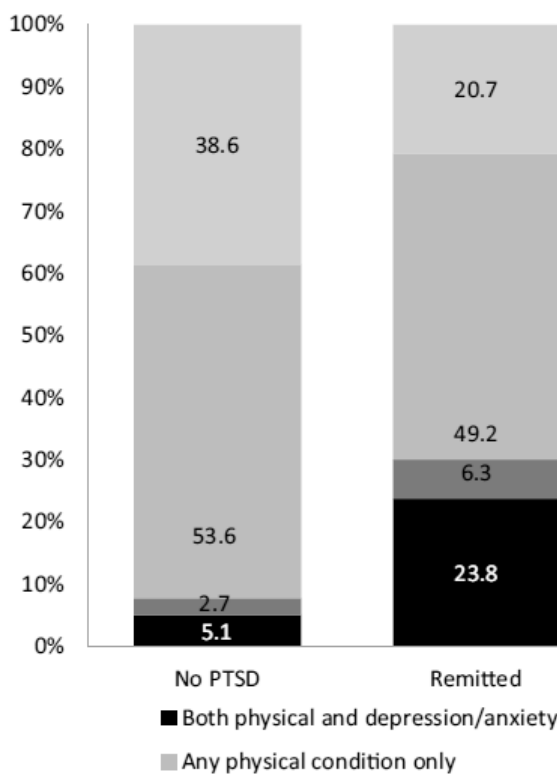

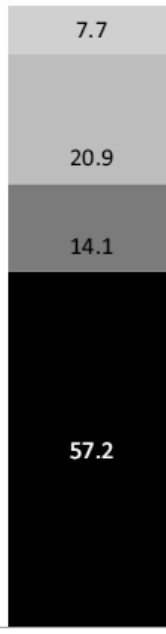

Delayed

Depression/anxiety only

No comorbidities

Figure 3. Age-adjusted prevalence of overall comorbidity status by PTSD-status (no PTSD: 23,213; remitted PTSD: 2,901; delayed PTSD: 2, 139; chronic PTSD: 1,749) (Li et al. 2018)

Longitudinal studies that follow up samples of people affected by recent severe traumatic exposures who present a new onset of PTSD in the aftermath present a favourable opportunity to assess physical morbidity associated more closely with courses of PTSD. More than 30.000 New York citizens responding World Trade Center Health Registry had been enrolled reporting no pre-9/11 PTSD and were followed up in successive waves of investigation over a period of ten years. PTSD trajectories (chronic, delayed, remitted, no PTSD) were identified and data on both psychiatric and physical comorbidities were collected. At wave 3 (2011/2012) the following survey on the overall comorbidity dependent on PTSD trajectory showed (Figure 3).
The age-adjusted prevalence of comorbid mental and physical comorbidity was highest in chronic PTSD (68.6\%), followed by delayed PTSD $(57.2 \%)$, remitted PTSD (23.8\%), and no PTSD (5.1\%), whereas the ageadjusted prevalence of no comorbidity, in contrast, was highest in the no PTSD trajectory $(38.6 \%)$, and lowest in chronic PTSD (4.2\%). Prevalence of several indicators of poor health-related quality of life (poor health status; $\geq$ 14 unhealthy days; $\geq 14$ activity limitation days) measured at wave 3 was significantly amplified by these assessed comorbidities accordingly. The data underlined that both mental and physical comorbidities contributed equally, but independently to the severity of poor healthrelated quality of life (Li et al. 2018). 
Although the majority of empirical studies emphasized that it is first and foremost diagnostic status of PTSD after trauma exposure that is associated with an increasingly impaired somatic health (Schnurr 2015), trauma events per se may have negative somatic health consequences as well. A closer look at this association between potentially traumatic events and physical conditions is relevant as incidents of trauma exposure epidemiologically occur in a much higher frequency than eventually resulting posttraumatic psychiatric disorders, such as PTSD, depression, anxiety etc. and therefore present a major challenge to any health care system. Data from the South African Stress and Health Study on a populationrepresentative sample of 4.433 adults aged 18 and older provide an interesting insight into this issue (Atwoli et al. 2016). The cross-sectional investigation included a professional psychiatric interview by CIDI, systematically collected information on life-time exposure to 27 potentially traumatic events (PTE) grouped into seven trauma types (war events, physical violence, sexual violence, accidents, unexpected death of loved person, traumatic events occurring to a loved person, witnessing acts of violence), and measured 20 physical conditions clustered into five categories (cardiovascular, arthritis, respiratory, chronic pain, and other conditions) for previous year incidence. After adjusting for sociodemographic variables and psychiatric disorders, respondents with any potentially traumatic events prior to the onset of any physical condition presented increased odds ratios of all assessed physical conditions ranging between 1.48 for arthritis and 2.7 for chronic pain in comparison to those participants reporting no PTE. Sexual and physical violence, unexpected death of or traumatic events occurring to a loved person increased the odds of nearly all physical conditions. There was a clear dose-response relationship between the number of trauma exposures and increased odds of all physical conditions. The study emphasized that traumatic events may indeed confer proper risks to a broad spectrum of eventually chronic physical conditions independent of posttraumatic stress disorders and, in addition, that each cumulative PTE exposure is increasing these risks further (Figure 4).

Posttraumatic physical health not only refers to somatic symptoms in the context of medically welldefined, physician-diagnosed somatic diseases, but also to a great many bodily complaints, symptoms, syndromes or disorders for which no or no sufficient organic causes can be found. These traditionally called "symptoms of somatization" or "somatoform symptoms" have also frequently to be considered within a posttraumatic context (Sack et al. 2007; Gupta 2013; Pacella et al. 2013). A recently published study reported on civilian war survivors from Kosovo ten years after the war (Morina et al. 2018). A random sample of 142 adult civilians aged $\geq 18$ years was enrolled from the general population. The participants had to confirm an exposure to at least one war-related PTE while living in one of three different regions of Kosovo known as former active war zones. Exposure to various PTEs was assessed according to a combined trauma event list of 23 items (Harvard Trauma Questionnaire, first part of Posttraumatic Diagnostic Scale - PDS). Severity of the 17 PTSD symptoms according to DSM-IV was measured by PDS that also allowed a categorical diagnosis of probable PTSD. Frequency of each PTSD-symptom in the previous month was rated on a 4-point scale ranging from $0=$ "never" to $3=$ " 5 times per week or more/nearly always", yielding a current total severity score ranging from 0 to 51 . Somatic symptoms were assessed by the 12-item somatization subscale of the Symptoms Checklist-90-revised (SCL-90-R). The main findings are presented in Figure 5:

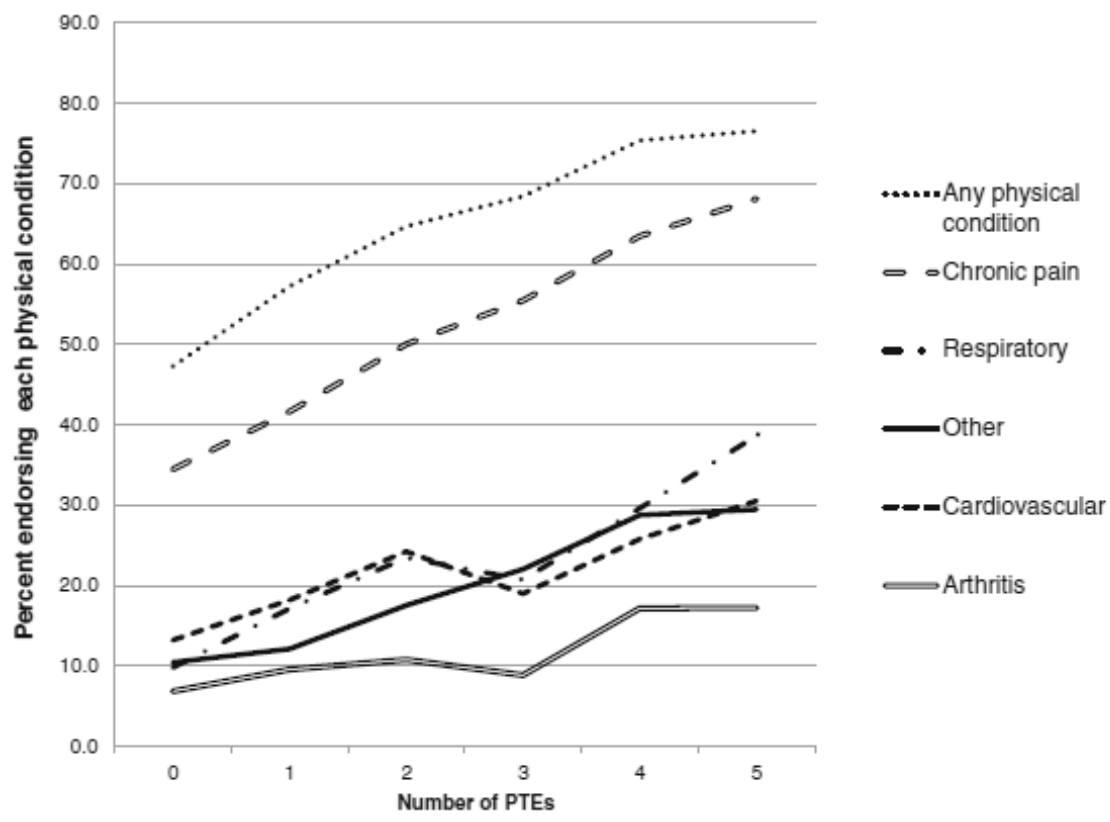

Figure 4. Percentage of study participants reporting physical conditions by the number of cumulative PTEs reported (Atwoli et al. 2016) 


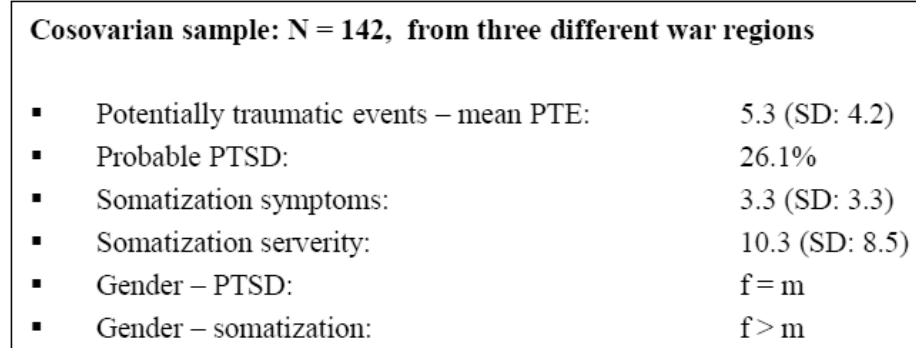

Somatization severity and symptom count by PTSD diagnosis

\begin{tabular}{llllll} 
& \multicolumn{2}{l}{ PTSD $(\mathrm{n}=37)$} & no PTSD $\left(\begin{array}{l}\mathrm{n}=105) \\
\mathrm{M}\end{array}\right.$ & $\begin{array}{l}\mathrm{M} \\
(\mathrm{SD})\end{array}$ & t-test \\
\hline Somatization severity & 15.9 & $(8.8)$ & 8.3 & $(7.5)$ & $5.104^{* * *}$ \\
Somatization Symptoms & 5.65 & 3.38 & 2.57 & 2.29 & $4.130^{* * *}$ \\
\hline
\end{tabular}

Somatization: subscale Somatization of SCL-90-R;

Diagnosis of probable PTSD by Posttraumatic Diagnostic Scale

Figure 5. Trauma exposure and the mediating role of posttraumatic stress on somatic symptoms in civilian war victims (Morina et al. 2018)

Civilian war survivors had experienced or witnessed a mean of 5.3 potentially traumatic events. The rate of diagnosis of probable PTSD in this random sample was $26.1 \%$. The severity score for somatization symptoms measured by the somatization subscale of SCL-90-R was 10.3. An average of 3.3 different somatization symptoms was reported. Women showed higher levels of somatization symptoms than men, whereas the levels of PTSD symptoms were similar in men and women. Participants with the diagnosis of probable PTSD presented higher scores both in somatization symptoms and somatization severity. In a statistical mediation analysis posttraumatic stress mediated the negative impact of trauma exposure in a significant way accounting for $37 \%$ of the variance in somatization symptoms (Morina et al. 2018).

\section{Intricate association between trauma exposure, posttraumatic stress syndromes and somatic health status - general focus on somatization symptoms/syndromes}

Medically unexplained symptoms, somatoform disorders/somatic symptom and related disorders, and functional bodily distress syndromes define a major challenge at every level of medical care. Doctors and patients meet equal difficulties in understanding and dealing with somatic symptoms that as a rule transcend narrowly defined categories of physical diseases. Complaints and symptoms of so-called "somatization" behaviour may not only emotionally strain physicianpatient relationship and be associated with a high utilizing and often negatively self-perpetuating illness behaviour but may also frequently run a chronic course of illness resulting in manifold psychosocial disabilities and a significantly reduced health-related quality of life (Creed et al. 2011). The conceptualization of these somatization symptoms/ syndromes has followed diverging diagnostic traditions within psychiatry and psychotherapy on the one side and clinical medicine and medical subspecialties on the other. The diagnostic challenge is underlined by the very clinical fact that these symptoms, syndromes and disorders of "somatization" seem to be lying somewhere in a puzzling triangle between anxiety and stress-related disorders, depressive disorders and functional disturbances of various organ systems. From a perspective of aetiopathogenesis modern psychiatric conceptualization principally refers to a central role of stress/distress and its impact on perceptive, affective, cognitive, behavioural and physiological compartments (Kapfhammer 2017b). It emphasizes variable biopsychosocial influences as possible predisposing, precipitating and perpetuating etiological factors at successive stages of the course of illness. A conceptual model is presented in Figure 6.

Numerous epidemiological and clinical studies underline that at least a subgroup of persons from general population or of patients in various clinical samples present symptoms/ syndromes of somatization that have to be considered in a posttraumatic context due to either adverse events during early childhood or/and to current trauma exposure (Maaranen et al. 2005, Roleofs \& Spinhoven 2007, McFarlane et al. 2008). A strong association between conditions of chronic pain on the one hand, and trauma exposure and posttraumatic stress disorders on the other is well established (Siqveland et al. 2017). 
This special link seems to be even more pronounced in clinical samples of patients with functional neurological disorders (conversion and dissociative disorders) (Roleofs \& Pasman 2016). This is also true for various functional somatic syndromes defined within medical subspecialties (e.g. fibromyalgia, chronic fatigue syndrome, irritable bowel syndrome). A systematic review and metaanalysis confirmed that individuals who reported exposure to trauma had an increased odds ratio of
2.7 regarding various functional somatic syndromes. The magnitude of the association with PTSD was significantly stronger than with trauma exposure alone (e.g. sexual, physical abuse). This PTSD-related effect was strongest after military combat exposure. Chronic fatigue syndrome had a larger association with reported trauma than fibromyalgia or irritable bowel syndrome. This association seemed to be stronger in men than in women (Afari et al. 2014; Figure 7).

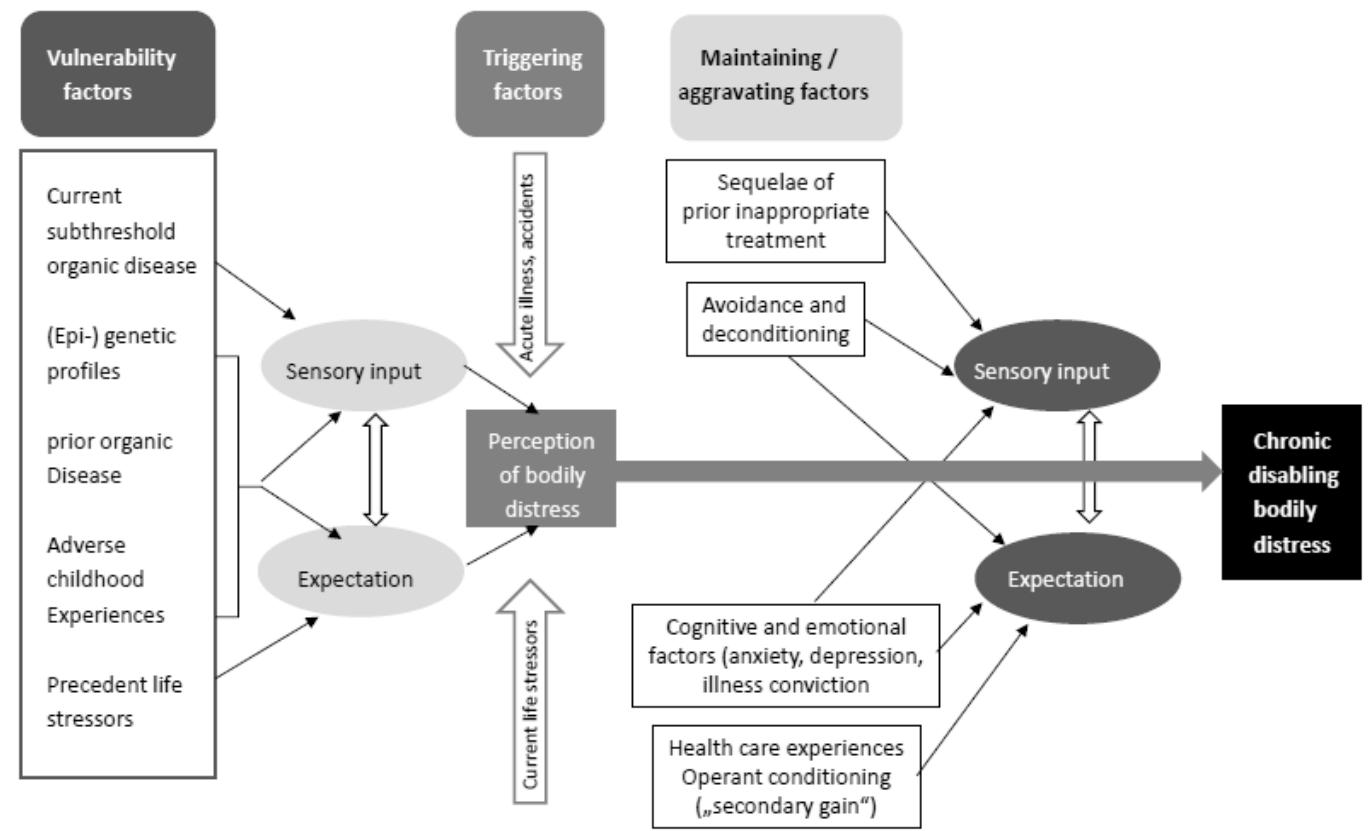

Figure 6. Conceptual model of somatization by predisposing, eliciting, and maintaining factors in the course of illness (Henningsen et al. 2018)

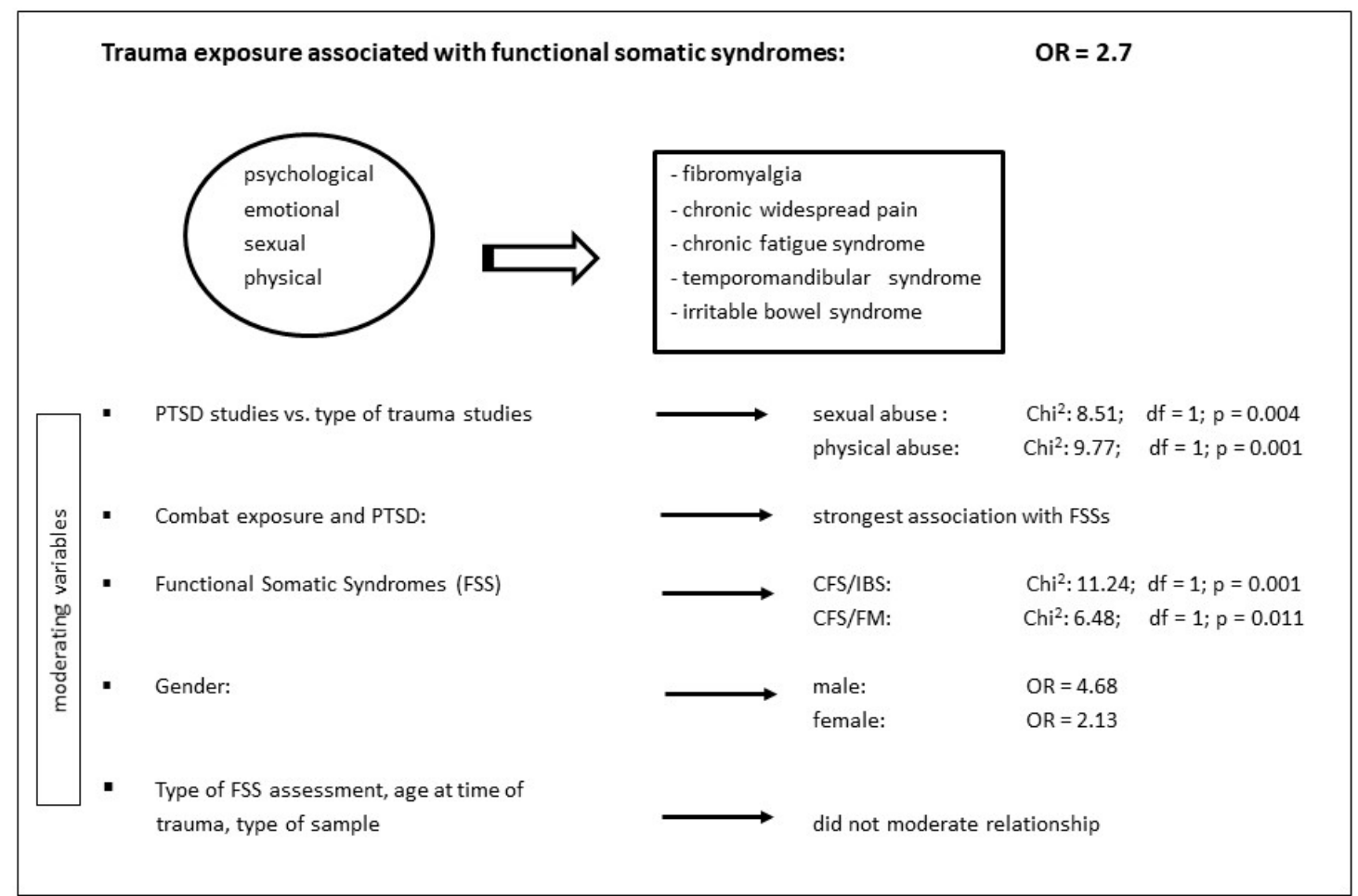

Figure 7. Psychological trauma and functional somatic syndromes: A systematic review and metaanalysis (Afari et al. 2014) 


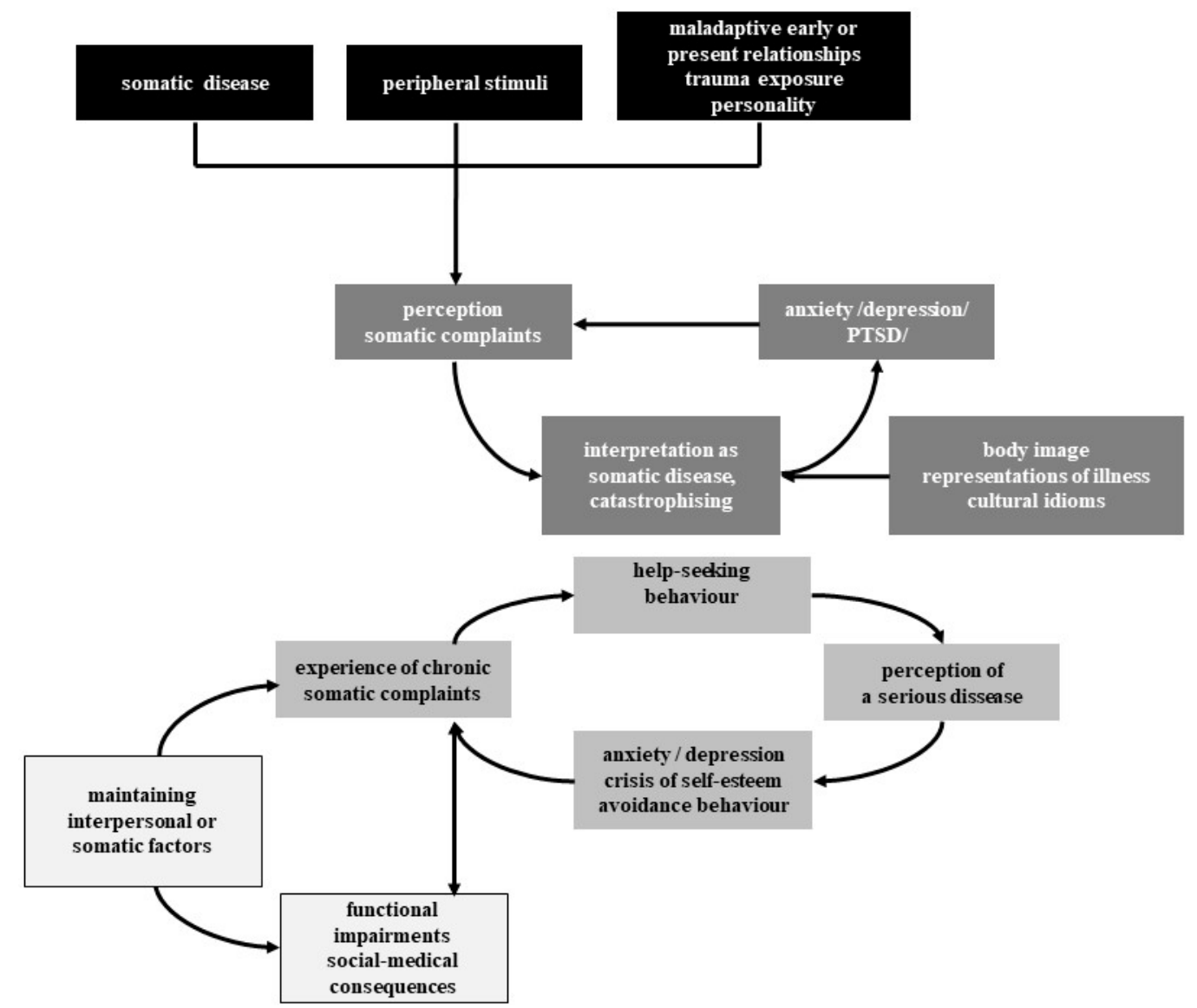

Figure 8. Multifactorial model of somatization during course of illness (Henningsen et al. 2007)

\section{How may we understand this significant association between trauma exposure, posttraumatic stress disorders and increased rates of somatization symptoms/syndromes?}

As a matter of course, not all patients presenting with symptoms and syndromes of somatization do have a history of past or current trauma exposure. Traumatic events and subsequent posttraumatic stress disorders may have a special impact on triggering and maintaining somatic symptoms and simultaneously on attentional, emotional and behavioural systems. Trauma-related effects have to be considered, however, as interacting with a great many other biopsychosocial factors that may not be trauma-related and may also be highly variable during various stages of the course of illness (Figure 8).

Regarding the various PTSD symptom clusters there is good empirical evidence that it is first and foremost the cluster of autonomous hyperarousal mediating strong somatic reactions during and in the aftermath of trauma exposure (Gupta 2013, Morina et al. 2018). It is not only a prevailing sympathetic hypertonus alone leading to well-known symptoms of painful muscle tension in localized or widespread body regions, hyperventilation, increased blood pressure and heart rate, abnormal endocrine and immunological reactions. It is more a general imbalance between the sympathetic and parasympathetic branches of the autonomous nervous system that seems to be an overarching characteristic (Sack et al. 2004). From a perspective of somatization a transitional or persisting parasympathetic hyperarousal is associated with rather a different pattern of somatic symptoms, e.g. bradycardia, gastrointestinal disturbances, altered processing and perception of pain signals, motor blockades, and general physical inactivation (Porges 2009). It is actually frequent cycling between sympathetic and parasympathetic dominance in the immediate aftermath of trauma exposure that seems to deliver a decisive impact on the early sensitization of trauma memory and associated physiological systems (Antelman et al. 1997). In the further course of illness significant alterations both in neuroendocrine and immunological/ inflammatory systems may perpetuate a process of central sensitization of the various neurobiological systems of stress regulation maintaining chronic somatization, particularly in states of chronic pain and fatigue (Rief et al. 2010, Pace \& Heim 2011, Morris et al. 2017, EllerSmith et al. 2018). This pathophysiological process of central sensitization may be even more strengthened by frequent other comorbid psychiatric disorders, such as depressive and anxiety disorders. On a psychological level any trauma-related somatic hyperarousal is typically coupled with a perceptive amplification of physical sensations and a prevailingly body-focussed and often catastrophizing style of affective and cognitive processing (Rief \& Broadbent 2007, Kapfhammer 2017b). 

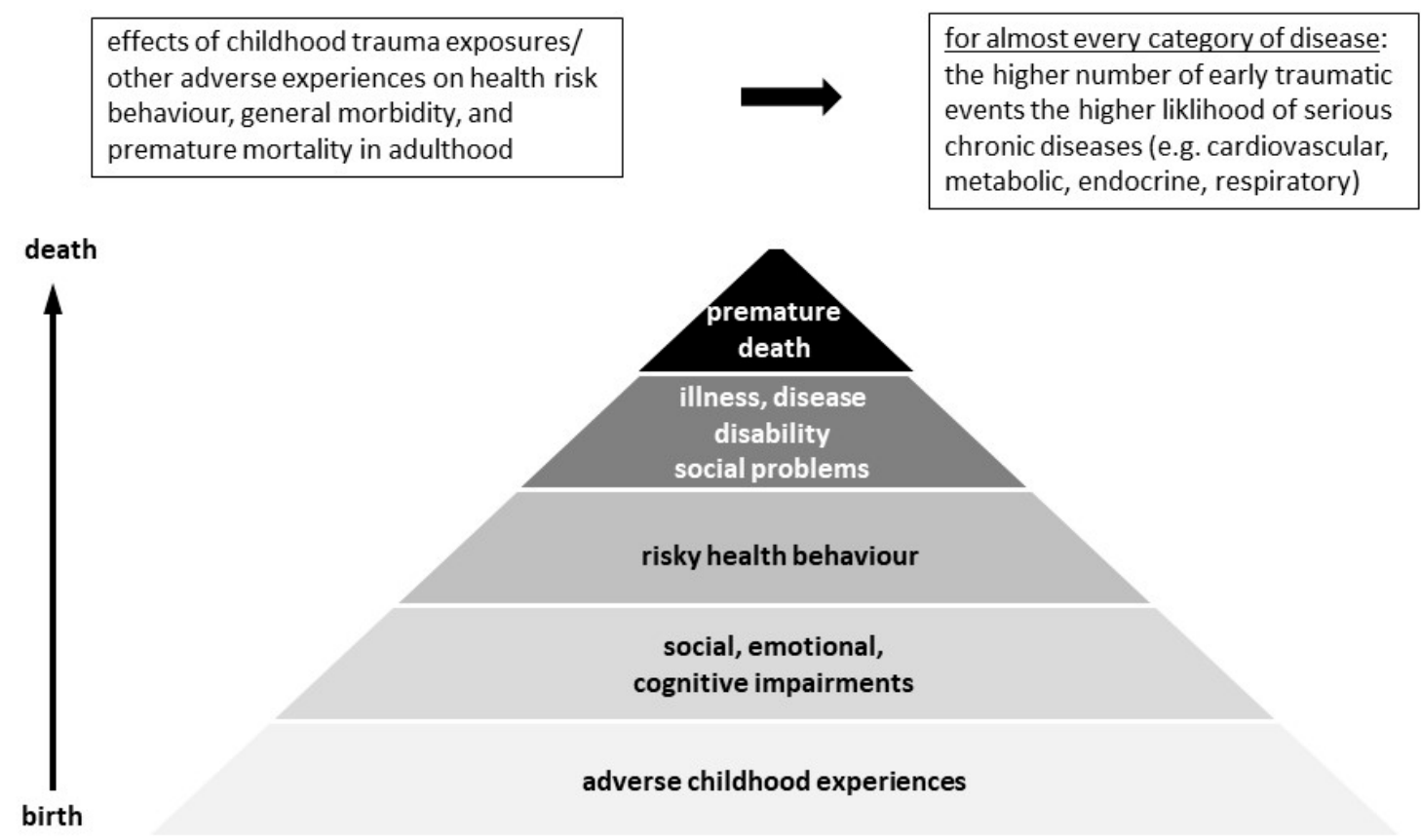

Figure 9. Relationship between childhood adverse events and health status in adulthood (According to: Felitti et al. 1998)

In respect of functional neurological disorders, also described as variants of conversion or dissociative disorders, clinical phenomenology seems to be somewhat different and predominantly characterised by qualitative alteration or even by complete loss of basic attentional, motor and sensory neurological functions. Pathways mediating effects of trauma exposure and posttraumatic stress disorders on these functional neurological disorders are best described and conceptualized within complex models of dissociation (Gershuny \& Thayer 1999, Nijenhuis 2000, van der Hart et al. 2004). Trauma-based models of dissociation not only allow a favourable insight into a diverse functional neurological phenomenology, but are especially compatible with underpinning neurobiological processes that may be delineated by neurophysiological or neuroimaging methods (Brown 2016, Roloefs \& Pasman 2016, Kapfhammer 2017c, 2018).

On a psychosocial level, trauma-based syndromes of somatization are disproportionately associated with high-utilizing illness behaviour in medical and social services, high risk of self-induced or iatrogenic harm, severe social disabilities and significantly reduced health-related quality of life (Katon et al. 2001, Sansone et al. 2005, Creed et al. 2011, Kapfhammer 2017b).

\section{Intricate association between trauma exposure, posttraumatic stress syndromes and somatic health status - general focus on comorbid physical diseases}

In their landmark Adverse Childhood Experiences (ACE) Study Felitti and co-authors (1998) impressively demonstrated a strong graded relationship between the breadth of exposure to childhood emotional, physical, or sexual abuse, and household dysfunction during child- hood on the one hand and multiple health risk behaviours, a great many mental and physical diseases and increased rates of premature mortality in adulthood on the other (Figure 9).

Meanwhile available knowledge based on a great deal of following empirical studies has impressively confirmed Felitti's findings. A recent systematic review and meta-analysis found that individuals with $\geq 4$ ACEs were at increased risk of all mental, physical and psychosocial health outcomes compared with individuals with no ACEs. Associations were weak or modest for physical inactivity, overweight or obesity, and diabetes (ORs of less than two); moderate for smoking, heavy alcohol use, poor self-rated health, cancer, heart disease, and respiratory disease (ORs of two to three), strong for sexual risk taking, mental ill health, and problematic alcohol use (ORs of more than three to six), and strongest for problematic drug use and interpersonal and self-directed violence (ORs of more than seven) (Hughes et al. 2017). The epidemiological literature with a primary focus on PTSD and defined physical comorbidities as long-term-sequelae in the aftermath of adverse/traumatic events has also underlined significant associations with a broad spectrum, including cardiovascular disease, diabetes mellitus, gastrointestinal disorders, some types of cancer, and all-cause or specific premature mortality (Gradus 2017).

\section{How may we understand this significant association between trauma exposure, posttraumatic stress disorders and increased rates of comorbid physical diseases?}

As a matter of course again, any linear or even monocausal approach to analyse the pathways from trauma exposure to posttraumatic stress disorders to increased 
somatic health risks and finally to heightened rates of comorbid somatic diseases and premature mortality is obsolete. As Schnurr $(2014,2015)$ has emphasized in several important papers on the issue, a complex strategy of analysis has to be utilised to elucidate the many interacting factors contributing to trauma-related somatic comorbidities (Figure 10). She underlined that only a minority of traumatized patients directly acquired enduring somatic illness conditions or had sustained physical injuries from external traumatic events, such as military combat trauma. She commented that "the types of health problems that emerge typically are not linked to the type of trauma experienced" (Schnurr 2015, p. 89). The most consistent and primary mechanism seems to be associated with the diagnostic status of PTSD or other comorbid mental disorders such as depressive, anxiety or substance use disorders that all may implicate severe and persistent distress. This major pathway may be determined by PTSD-related attentional (amplification of sensory perception), affective and cognitive (trauma- related alterations in cognitions and mood), and behavioural (smoking, substance use, poor self-care, poor diet, lack of physical activity, reduced adherence to medical regimens) mechanisms, in tight interaction with various psycho- and neurobiological mechanisms underpinning the complex pathophysiology of PTSD.

From a theoretical perspective this multifaceted model of analysis has to be transposed to a longitudinal developmental line that starts with various predisposing, precipitating and perpetuating risk factors and finally indicates increased rates of somatic morbidity and mortality. In an earlier paper Schnurr and Green (2004) had leaned upon the concept of allostatic load to stress this point of view (Figure 11). As McEwen and Stellar (1993) had explicated this concept: "the strain on the body produced by repeated up and downs of physiologic response, as well as the elevated activity of physiologic systems under challenge, and the changes in metabolism and wear and tear on a number of organs and tissues" (p. 2094).

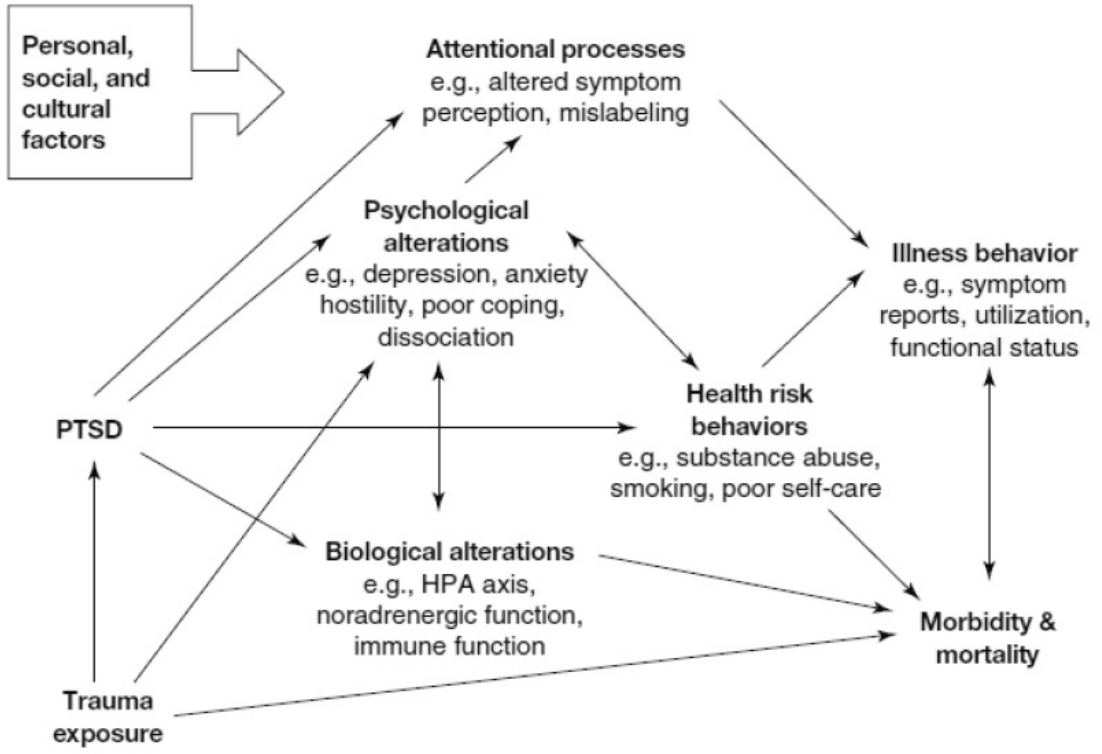

Figure 10. Model relating trauma exposure, PTSD to physical health outcomes (Schnurr 2015, p. 89)

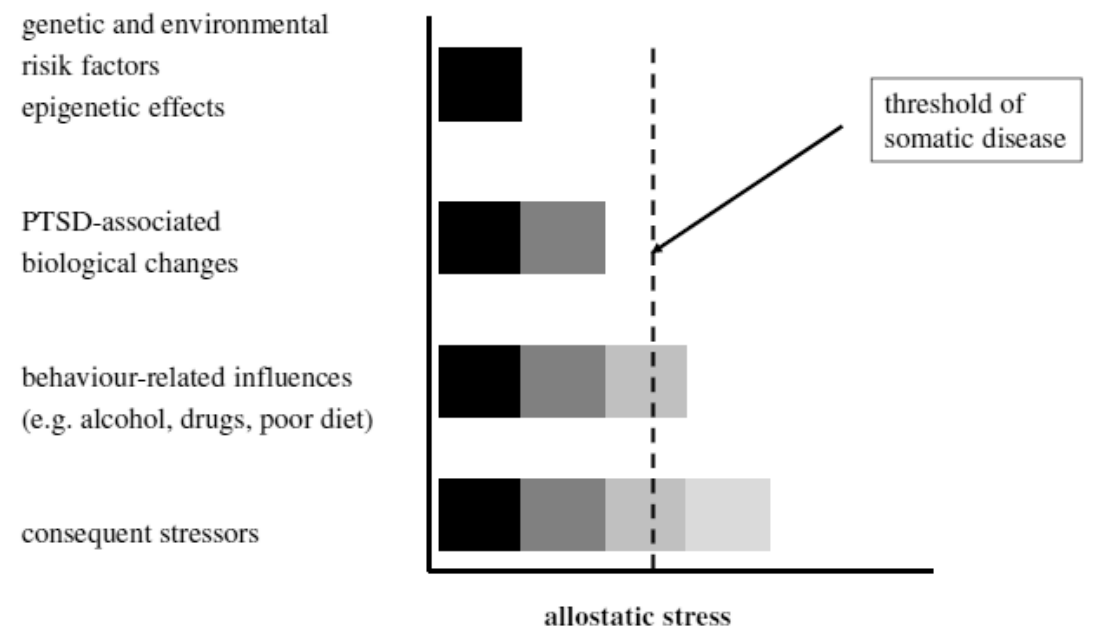

Figure 11. Pathogenetic focus on PTSD-associated physical morbidity according to concept of allostatic load (Modified: Schnurr \& Green 2004) 


\section{Exemplary focus on increased risks of cardiometabolic /cardiovascular diseases as sequelae of trauma exposure and posttraumatic stress disorders}

A short overview shall be presented in some very broad lines for matters of illustration. There is indeed a strong meta-analytic association between PTSD and increased risk for early incident cardiovascular disease and cardiovascular mortality. This effect is beyond the one mediated by depression that is comorbid to chronic PTSD so frequently (Edmondson et al. 2013, Edmondson \& von Känel 2017). In the following summary sections one has generally to keep in mind that each focus on a special topic may hint either to preferentially acute effects or to predominantly enduring processes that successively contribute to a condition of increasing allostatic burden. And one has always to consider an intricate network of interrelationships and interactions between the various levels of investigation.

\section{Genetics, epigenetics, and risks of cardiometabolic/ cardiovascular diseases}

Current research data have convincingly demonstrated that multiple molecular genetic polymorphisms have an impact on the risk of PTSD after adverse events and trauma exposures both in early childhood and later in life (Teicher et al. 2016, Wolf \& Schnurr 2016a). These genetic polymorphisms may mediate their effects via functional alterations in multiple neurotransmitter systems (serotonergic: 5-HT2A,5-HTTLPR; dopaminergic:
DAR2, DAT, Dopamin- $\beta$-Hydroxylase; noradrenergic: neuropeptide Y, COMT (Val158Meth); GABA-ergic: GABA-A-R $\alpha 2$, $\alpha 6$, GABA-A-R $\beta 3$ ), HPA-axis (GCR; FKBP5; CRHR1), neuropeptides (apolipoprotein, brainderived neurotrophic factor - BDNF, estrogen-sensitive pituitary adenylate cyclase-activating peptide - PACAP) and various immune/inflammatory system-related processes (e.g. single nucleotide polymorphism of CRP) (Domschke 2012, Michopoulos et al. 2015, Zass et al. 2017). Major gen-environment interactions may shape life-long online genetic expression activities by epigenetic mechanisms (e.g. DNA-methylation, posttranslational histone modification, small RNA signalling, chromatin conformation changes), either blocking or activating circumscribed DNA sequences, and thus causing altered functions of HPA-axis, neurotransmitter systems or/and immune/ inflammatory systems underpinning the complex pathophysiology of PTSD (Sheerin et al. 2017, Daskalakis et al. 2018; Figure 12).

As regards heightened cardiometabolic/cardiovascular risks in the aftermath of trauma exposure and PTSD in particular, modern genetics and epigenetics have indicated a major genetic overlap between stressrelated and mood disorders and cardiometabolic diseases (Amare et al. 2017, Sumner et al. 2017). Several candidate genes identified within the realm of either mental disorder or physical disease show also pleiotropic effects regarding the comorbid other disorder/ disease, respectively, thus underlining some shared common pathways (Figure 13).

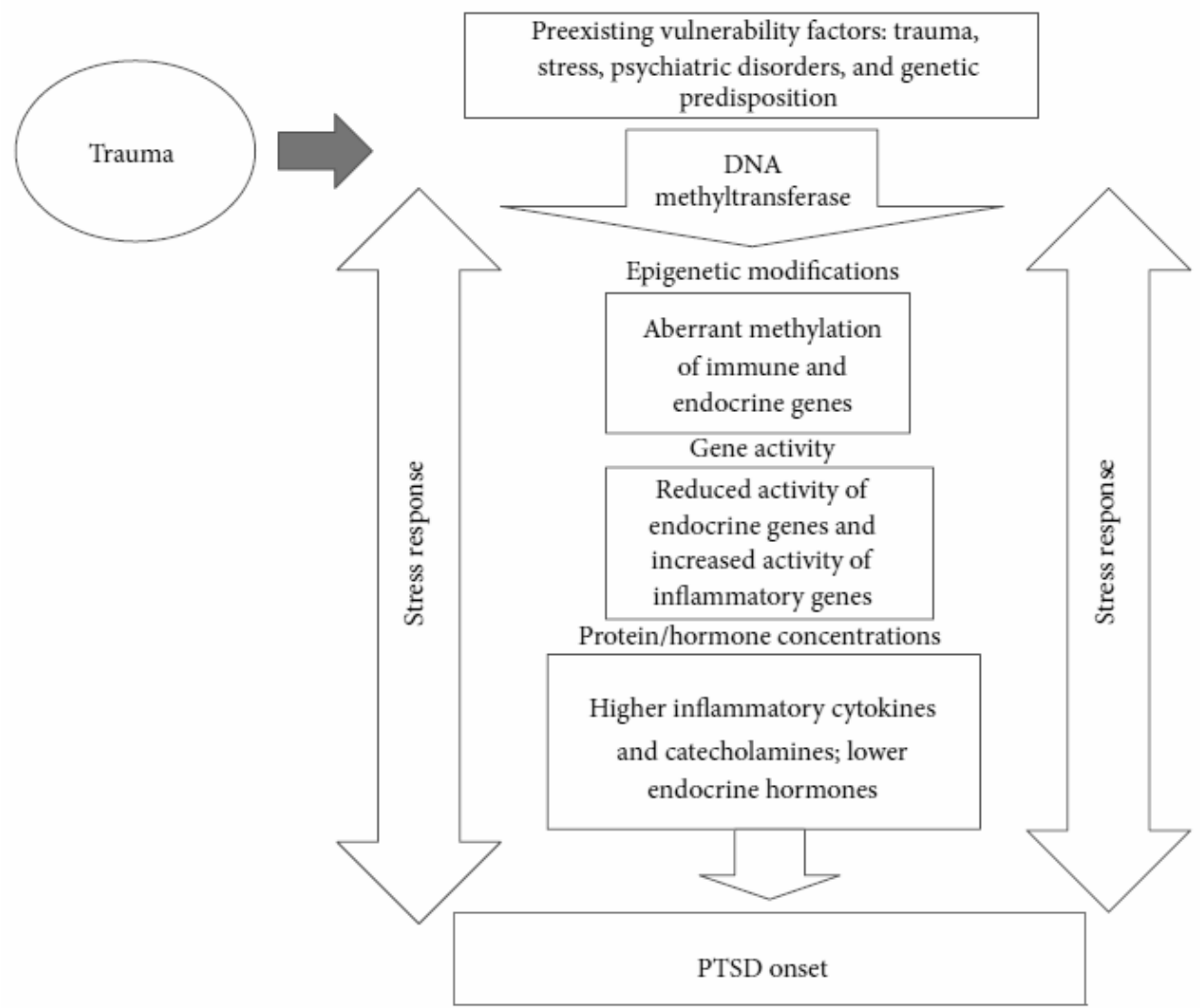

Figure 12. Epigenetic predisposition and risk of PTSD after trauma exposure in adulthood (Heinzelmann \& Gill 2013) 


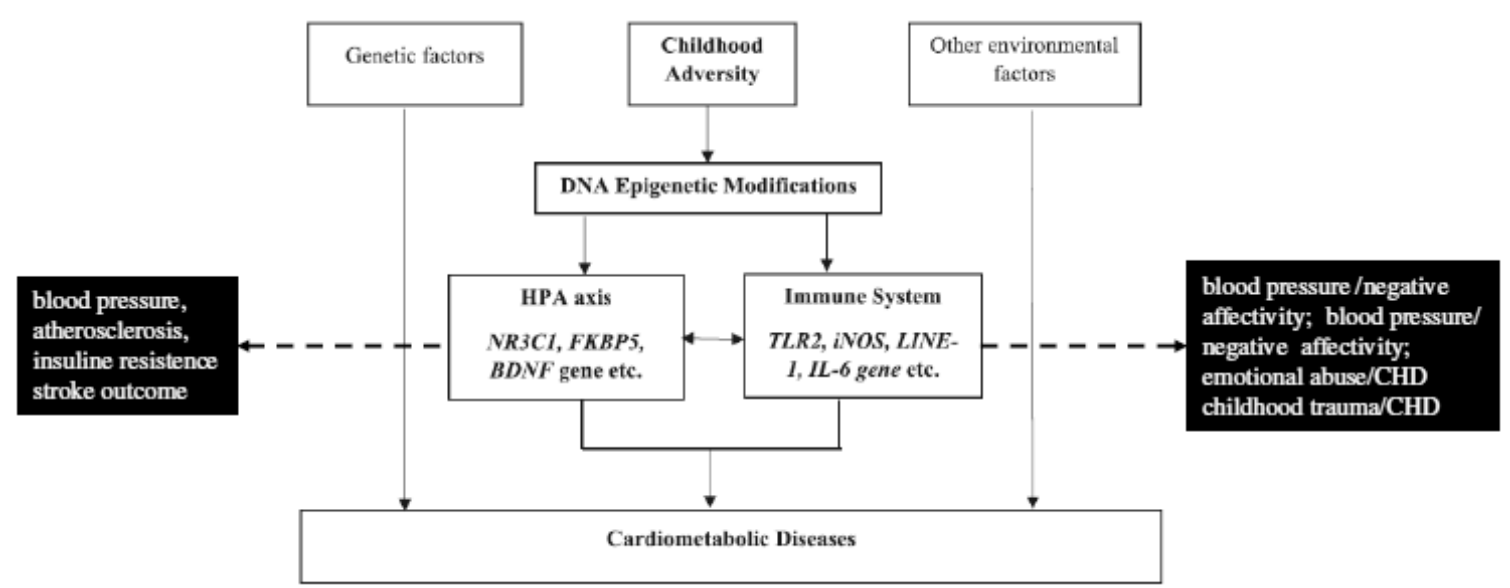

Figure 13. Role of DNA methylation in association between childhood adversity and cardiometabolic disease (Modified: Hao et al. 2018)

\section{Behaviour- and lifestyle-based intermediate clinical conditions as proximal risk factors of cardiometabolic/cardiovascular diseases}

As has been outlined already, chronic courses of PTSD are frequently characterized by various persisting emotional, cognitive and behavioural patterns that may increase further individual suffering and overall allostatic load. Each unfavourable pattern may not only maintain the psychological and psychosocial burden of PTSD, but may also contribute to successively worsening somatic health conditions in general, and may exacerbate cardiometabolic/cardiovascular health risks in particular. Some of these PTSD-related patterns are strongly associated with empirically well validated, "classical" risk factors of cardiometabolic/ cardiovascular disease, such as metabolic syndrome (hypertension, obesity, dyslipidaemia, insulin resistance/elevated fasting glucose), or type II diabetes mellitus. This equally applies to prevailing negative affective states such as panic and anxiety, depression and demoralisation, anger and hostility, to enduring disadvantageous personality traits or social behaviours such as D-personality (combination of negative affectivity and social inhibition), social isolation, stress-inducing low job performance, and as well to a lot of health-risky behaviours such as physical inactivity, poor dietary habits, bad sleep hygiene, heavy smoking, alcohol or substance abuse, lack of motivation to change unhealthy lifestyles, to adhere to prescribed medication, consultations or rehabilitation programmes (Pedersen et al. 2017).

Taking this focus, it has to be considered a major health challenge, when, for example, the rate of hypertension has been nearly 2 -fold in individuals with vs. without PTSD in the National Comorbidity Survey (Kibler 2009), with similar results in a sample of more than 300.000 Afghanistan and Iraq war veterans (Cohen et al. 2009). In another, prospective study on some 200.000 veterans of recent US-military war deployments, over a median 2.4-year follow-up, the incident hypertension risk independently associated with PTSD ranged from hazard ratio, 1.12 to HR, 1.30. The interaction of PTSD and treatment revealed that treatment reduced the PTSD-associated hypertension risk from $\mathrm{HR}, 1.44$ for those untreated, to HR, 1.20 for those treated. The authors stressed that reducing the long-term health impact of PTSD and associated complications might actually require very early surveillance and treatment (Burg et al. 2017).

Carrying on this line of consideration, other components of the metabolic syndrome such as obesity finally contributing to a heightened risk of type II diabetes show a strong association to PTSD as well. A meta-analysis across nearly 10.000 PTSD patients and some 7.000 controls showed a pooled prevalence of metabolic syndrome of $38.7 \%$ in PTSD patients, with an increased $\mathrm{OR}=1.82$ compared to persons from the general population without PTSD (Rosenbaum et al. 2015). PTSD severity has been shown to be significantly associated with increasing risks of obesity, diabetes and cardiovascular disease. Both current PTSD and early life adversity have been found to mediate additive effects on these cardiometabolic outcomes (Farr et al. 2015). As most studies on the issue have been of a cross-sectional type, bidirectionality of causative effects cannot be ruled out by this epidemiological approach. Results from a longitudinal design investigation emphasized that PTSD severity predicted metabolic syndrome severity in follow-up, after controlling for initial metabolic syndrome severity, and not the other way round. Actually, persisting PTSD severity contributed to a significantly increased rate of metabolic syndrome diagnosis in the further course (Wolf et al. 2016). A recent study on patients using Veterans Health Administration for health care demonstrated that obesity moderated the association of PTSD and incidence of type II diabetes. The incidence of type II diabetes in not obese PTSD patients was, by contrast, similar to the US-national incidence rates. The results suggested that PTSD was not likely to have a direct causal association with incident type II diabetes. The authors discussed that future research was needed to determine whether PTSD remission could lead to loss of body weight and thus reduce incident type II diabetes (Scherrer et al. 2018). 


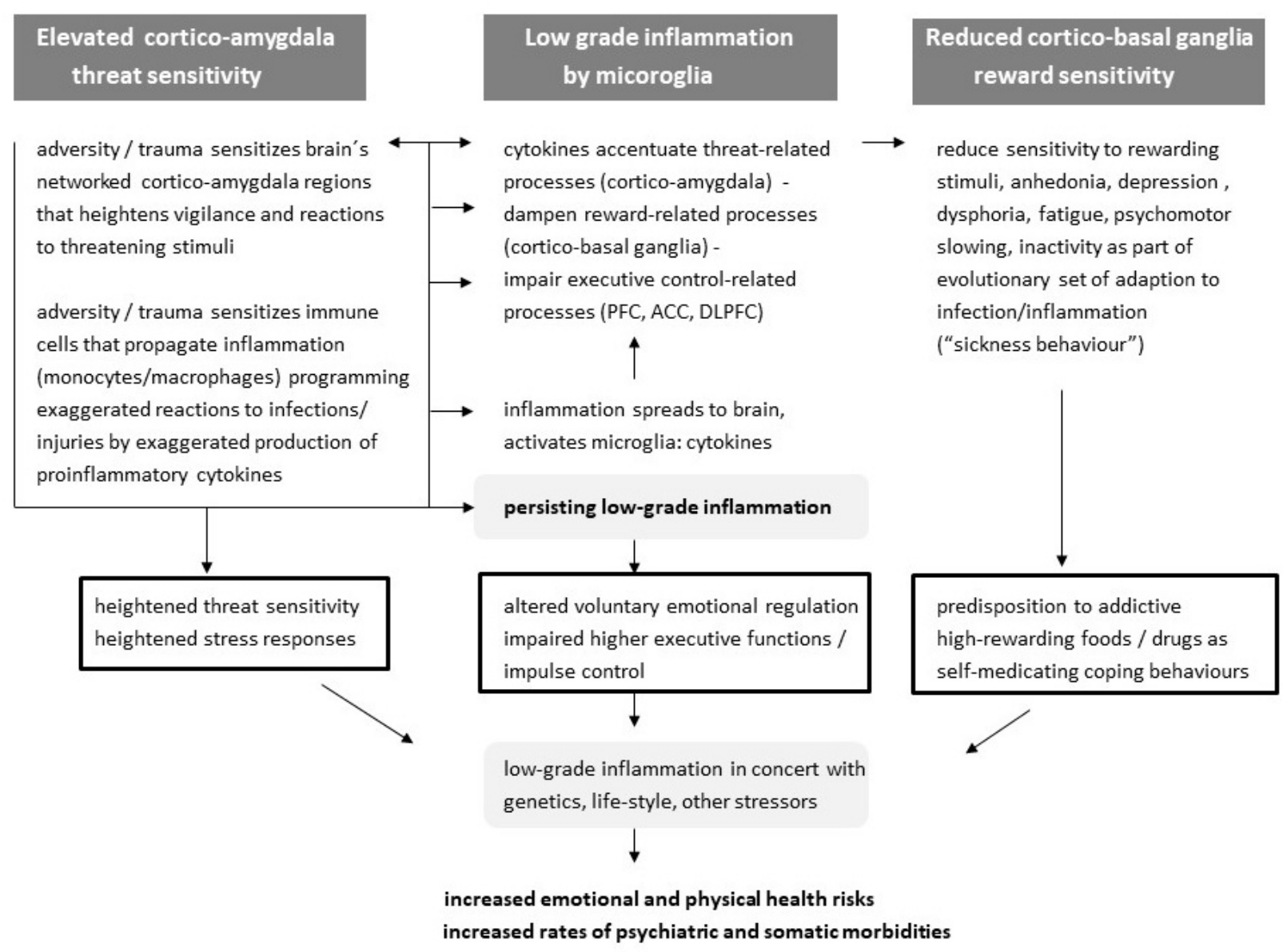

Figure 14. Effects of early-life adversity and later traumatic events on emotional and physical health across lifespan: a neuro-immune network hypothesis (Nusslock \& Miller 2016)

Nusslock and Miller (2016) suggested a stimulating model of analysing effects of early-life adversity on emotional and physical health across lifespan. Their "neuro-immune network hypothesis" underlined multidirectional interactions amongst a whole range of biological stress systems in the aftermath of trauma exposure and posttraumatic stress disorders. The heuristic framework pulled together empirical findings from the manifold domains that characterize the complex pathophysiology of trauma processing and PTSD. Its theoretical main focus was on trauma-/PTSD-related immunological /inflammatory changes. According to this model early-life adversity and also later traumatic events amplify crosstalk between peripheral inflammation and major neural circuitries subserving neurobiological threat-, reward-, and executive control-related processes. A resulting chronic low-grade inflammation is considered to contribute to obesity, insulin resistance, and other predisease states. Inflammatory mediators act on cortico-amygdala threat system, cortico-basal ganglia reward system, higher control and executive systems of the prefrontal cortex in a specific way that strongly predisposes traumatized individuals to certain behaviours that are meant to reduce stress or/and to cope with stress-related anhedonia and dysphoria, such as smoking, drug use, or consumption of high-fat / sugarrich diet, that, however, actually perpetuate the chronic course of PTSD. In concert with chronic inflammation these health-risky behaviours may accelerate emotional, cognitive and physical health problems furthermore (Figure 14).

As a matter of course, the authors'main focus on stress-triggered and then perpetuating conditions of chronic inflammation have to be widened and must appreciate the effects of highly interdependent other primary stress systems, such as HPA axis dysregulation and sustained autonomic imbalance (Wulsin et al. 2018). At this, it has to be considered that genetic variability and ongoing epigenetic processes have enduring impact of a variable kind on each neural circuit of these primary stress systems and also on intermediate behavioural phenotypes and clinical predisease states determined by these stress systems. And, "these genetic predispositions could also prime insufficient repair processes that lead the organism's failure to return to homeostasis" (Wolf \& Schnurr 2016 a, p. 8).

\section{Oxidative stress, mitochondrial dysfunction, inflammation, and neuroprogression indicating premature aging in chronic PTSD}

There has been emerging empirical evidence that increasing rates of early onset somatic comorbidities such as cardiometabolic/cardiovascular diseases and a specifically related mortality may be considered as part 
of a general process of premature aging in chronic PTSD (Lohr et al. 2015). Recurrent sensory-affective remembrances of trauma and concomitant activations of stress- and threat-related pathways may lead to persisting physiological hyperarousal and sleep disturbance. This stress-perpetuating syndrome may be characterized by elevated systemic levels of oxidative stress and inflammation that finally run in accelerated cellular aging and pathological remodelling of neurocircuits, i.e. neuroprogression (Miller et al. 2018). Oxidative stress is considered a cellular status that occurs when pro-oxidant molecules (e.g. reactive oxygen/ nitrogen species) exceed the counteractive capacity of anti-oxidants (e.g. glutathione, superoxide permutase). Oxidative stress is usually facilitated in a milieu of heightened proinflammatory activity, as is the case in PTSD with increased levels of circulating IL-6, IL-1 $\beta$, TNF $\alpha$, and interferon $\gamma$ (Passos et al. 2015). Persistent psychologic stress disrupts oxidant-antioxidant balance particularly within the brain, causing reduction in antioxidant enzyme function of glyoxalase (GLO)-1, glutathione reductase- 1 , manganese and $\mathrm{Cu} / \mathrm{Zn}$ superoxide dismutase. This eventually leads to glutathione depletion. Simultaneously interacting glutamate toxicity, calcium imbalance, and mitochondrial impairment collectively intensify oxidative stress finally leading to cell degeneration and apoptotic cell death (Salim 2017). Among human organ systems, brain is particularly vulnerable to oxidative stress due to its high energy and oxygen demand and its dense composition of oxidationsusceptible lipid cells. States of persisting oxidative stress are associated with disruptions of the blood-brain barrier, impaired neurogenesis, and altered brain morphology (Miller et al. 2018). Actually, persisting oxidative stress disturbs defined neurocircuitries, weakening and injuring hippocampal, amygdalar, and cortical connections, thus increasing symptoms of threat-related anxiety and depression on the one hand, and facilitating behavioural and cognitive deficits on the other. Oxidative stress-related damage to the brain has been shown to be significantly mediated by ALOX 12 and 15 gene activity transcribing the enzyme 12/15-lipoxygenase that both compromises mitochondrial integrity and produces pro-oxidant reactive oxygen species (Miller et al. 2015). Recently, Picard and McEwen (2018) underscored a central role of mitochondrial integrity within an overarching conceptual framework of psychological stress and evolutionary principles of adaption. Their model emphasized that any lasting mitochondrial dysfunction caused by states of psychological or/and physiological stress as paradigmatically processed in trauma and PTSD, may have far-reaching detrimental health consequences across lifespan. Mitochondrial defects directly affect the brain, the endocrine, and the immune system, all these altered systems contributing to an ever increasing allostatic load. Mitochondrial signalling has a major impact on gene expression and epigenetic modifications, and thus may profoundly modulate the rate of cellular aging.
One special molecular marker of accelerated general aging is indicated by a critical shortening of the telomere length. Telomeres are chromatin structures at the end of chromosomes. They are composed of repeat DNA sequences. Telomeres are considered to protect chromosomes from degradation and recombination. They are coated with a protein complex. Telomere length usually decreases with age. When its length becomes critically short, the risk of apoptosis increases. A great many studies have proofed that health-risky behaviours such as smoking, alcohol consumption, poor sleep hygiene, or age-related medical conditions such as cancer, diabetes, coronary heart disease are linked with a shortened telomere length. And this association has been repeatedly found also in major psychiatric disorders, such as in depression, schizophrenia, substance use, and not at last in PTSD (Lindqvist et al. 2015; Vakonaki et al. 2018). Wolf and Schnurr (2016b) consistently discussed the increased rates of comorbid early-onset cardiometabolic/cardiovascular diseases in the aftermath of traumatic events and posttraumatic stress disorders also within this broader context of stress-induced premature aging (Figure 15).

\section{How may we understand trauma- and PTSD- related effects on the pathophysiology of cardiometabolic/cardiovascular morbidity and on incident acute cardiac events?}

Epidemiogical literature clearly underscores that PTSD is to be considered an independent risk factor both for the development of cardiometabolic/cardiovascular morbidity and incident acute cardiac events such as myocardial infarction or sudden cardiac death. And PTSD-related effects surmount those cardiotoxic effects induced by states of depression, but frequently co-act synergistically with them. The multiple basic neurobiological mechanisms are to be sketched in a short summary (Brudey et al. 2015, Edmondson \& von Känel 2017, Esler 2017, Wirtz \& von Känel 2017, de Hert et al. 2018, Fleshner \& Crane 2018, Kivimäki \& Steptoe 2018, Mellon et al. 2018, Speer et al. 2018, Wulsin et al. 2018).

Within seconds after exposure to stress autonomous nervous system activates both sympathetic and parasympathetic responses. The catecholamines adrenaline released by the adrenal medulla and noradrenaline released by the sympathetic nerve terminals increase heart rate and decrease heart rate variability. Direct cardiostimulatory effects are positive chronotropy and inotropy mediated via $\beta 1$-adrenergic receptors, as well as pressor effects via $\alpha 1$-adrenergic receptors. There are close synergistic interactions with HPA-axis, immune/ inflammation system, and renin-angiotensin system. Sympathetic activity also significantly influences metabolic processes by facilitating lipolysis and affecting insulin sensitivity. Activation of the parasympathetic branch releases acetylcholine in order to counterregulate sympathetic overdrive. In states of persisting stress as in PTSD there predominates a sympathetic hyperarousal 


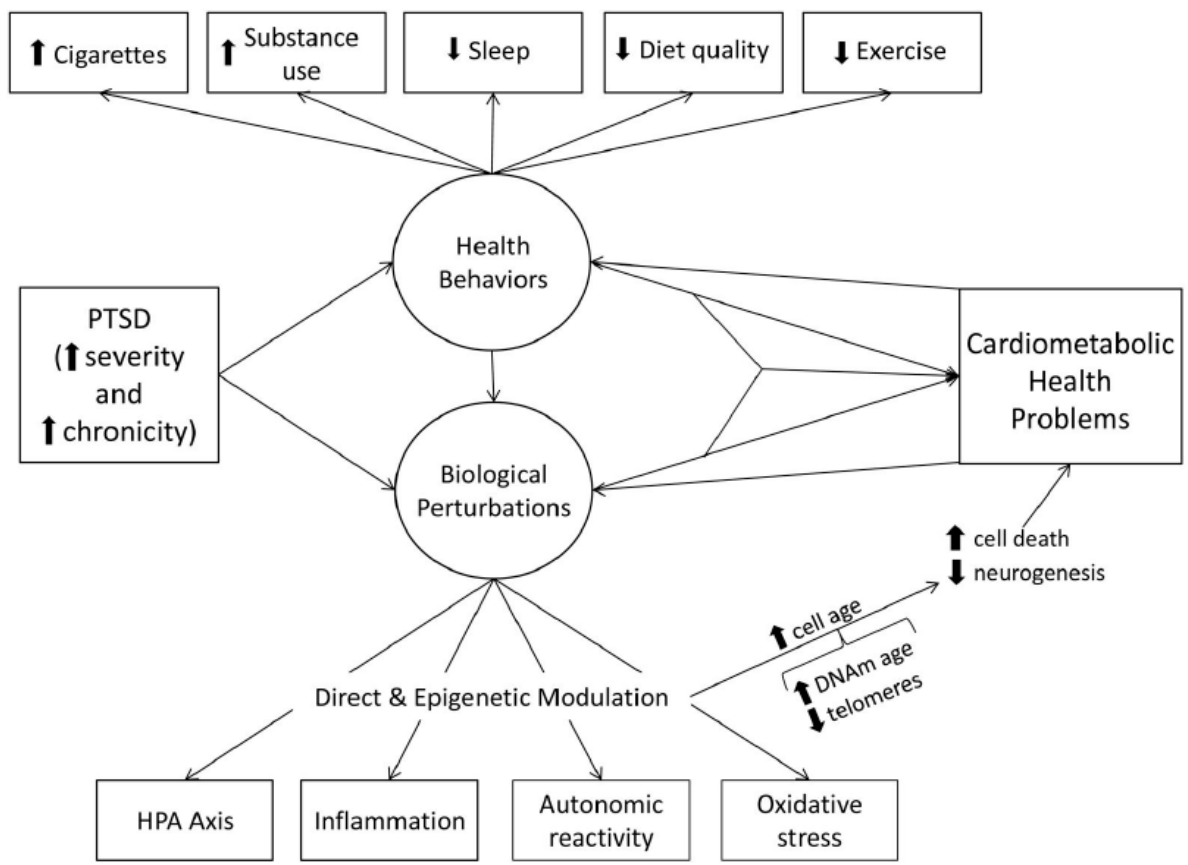

Figure 15. Conceptual model of hypothesized associations of PTSD and cardiometabolic/cardiovascular diseases via biological and health behaviour pathways (Wolf \& Schnurr 2016b)

with catecholamine spillover and withdrawal of parasympathetic inhibition. Typical pathophysiological features of sustained autonomous imbalance are heightened heart rate, increased QT-intervals in ECG, reduced heart rate variability, pre-ejection period, baroreflex sensitivity, and heart rate recovery after exercise or acute stress exposure. In particular reduced heart rate variability and reduced baroreflex sensitivity appears to be linked with carotid atherosclerosis, proinflammation and hypercoagulopathy. Increased QT-interval variability is considered a predictor of sudden cardiac death. In states of myocardial ischemia and ventricular instability any acute psychological stress may further lower the threshold of ventricular vulnerability period thus triggering chamber flicker, the probable mechanism of sudden cardiac arrest.

Within minutes after stress exposure HPA axis is activated via hypothalamic CRF, pituitary $\mathrm{ACTH}$, and final release of the glucocorticoid cortisol by the adrenal cortex. Cortisol increases the level of blood glucose, $\beta$ cells in the pancreas release insulin that fosters the transport of glucose from blood into fat tissues, muscles and liver. Another function of glucocorticoids is the mobilization and specification of immune responses during stress. Glucocorticoids show acute anti-inflammatory effects, i.e. they suppress the production and activity of pro-inflammatory cytokines. Autonomous nervous system and HPA axis co-act on haemostatic factors by increasing platelet activation, fibrinogen concentrations, blood viscosity and coagulation factors. PTSD-related HPA axis dysregulation includes lower basal cortisol levels and lower daily cortisol output, however, an exaggerated cortisol response to acute stress exposure at least in a subgroup of PTSD patients. Chronic HPA axis dysregulation may actually lead to a heightened state of pro-inflammatory activity. Under the conditions of reduced cortisol output or glucocorticoid receptor insensitivity the nuclear factor- $\mathrm{kB}$ in macrophages and T-cells is insufficiently controlled. Its disinhibition may lead to an exaggerated production and release of several pro-inflammatory cytokines such as IL-6, IL-1 $\beta$, TNF $\alpha$, and interferon $\gamma$ (see below). Chronic HPA axis dysregulation has been shown to be implicated in the development of heart failure and myocardial ischemia (see above).

Severe stress also shows a strong sterile inflammatory response. An evolution-based programme is initiated by first released catecholamines that activate overall circulating exosomes. Exosomes are small extracellular vesicles that express membrane adhesion molecules and immunostimulatory proteins, in particular so called DAMPs (danger-associated molecular patterns), thus starting a whole cascade of responses by immune-competent cells. Under condition of HPA axis dyregulation and sympathetic hyperarousal in PTSD a persisting lowgrade proinflammatory state may ensue. The negative mental and physical consequences may be manifold. Increased levels of IL-6, IL-1 $\beta$, TNF $\alpha$, interferon $\gamma$, CRP, white blood cell account, and adhesion molecules characterize this condition of chronic low-grade inflammation. Proinflammatory cytokines are able to cross the blood-brain barrier and can activate brainendogenous production of cytokines by microglia, thus contributing to further HPA axis dysregulation, hyperglutamatergic excitation, increased oxidative stress, and significant changes in various neurotransmitter systems. One decisive mediating mechanism is the kynurenine pathway that via activation of 2,3 indolamine-dioxygenase causes tryptophan depletion. Eventually overall lowered levels of serotonin, melatonin, and dopamine 
on the one hand, and heightened glutamatergic neurotransmission on the other underpin typical psychopathological features of PTSD, foster frequent comorbid depressive and anxiety disorders via induction of "sickness syndrome", and lead to neuroprogression via increased oxidative stress.

Chronic low-grade inflammation may also support the development of metabolic syndrome, can lead to obesity, insulin resistance, dysregulation of carbohydrate, lipid and amino acid production and utilization. Increased visceral fat tissue itself may further contribute to proinflammation via adipokines. The overall consequence may be escalating oxidative stress, mitochondrial damage, and premature aging.

Both chronic HPA axis dysregulation, sustained imbalance of autonomous nervous system, and enduring low-grade inflammation in chronic PTSD contribute to an accelerating process of atherosclerosis. Both increase in blood pressure and altered balance of vasoconstricting vs. vasodilating substances directly act on the endothelium of the heart arteries. Endothelial dysfunction is actually considered a key early contributor to cardiovascular risk. Insufficient flow-mediated vasodilatation, reduced production and bioavailability of nitric oxide (NO) on the one side, increased levels of endothelin-I, a particularly potent vasoconstrictive peptide, and von Willebrand factor on the other are characteristic features. Under conditions of increased cardiovascular demand endothelial dysfunction leads to major sheer stress on arterial walls, to heightened inflammatory responses, and to activated prothrombotic processes. This prothrombotic state may be characterized by increased concentration of fibrinogen, clotting factors (e.g. clotting factor 4 , $\beta$-thromboglobuline), fibrin D-dimer, plasminogen activator inhibitor-I, and activated thrombocytes. This highly interwoven complex of altered endothelial, inflammatory, haemostatic and prothrombotic components determines a slowly proceeding formation of atherosclerotic plaques. It is, however, haemodynamically induced erosion or rupture of an atherosclerotic plaque that finally causes thrombotic coronary occlusion, mediating an incident acute cardiac event. And this acute cardiac event may have started with an acutely triggered emotional state, such as PTSD-related panic, intrusive trauma remembrance or anger outburst.

\section{Concluding remarks}

In summary, empirical evidence underscores that trauma exposure, and in particular PTSD is significantly associated with major physical health problems in addition to well-known PTSD-related psychological, behavioural, and psychosocial impairments. Both self-reportbased and objective assessments emphasized significantly increased rates of somatoform/functional syndromes and physical comorbidities, premature all-cause and specific mortality rates, heightened medical utilization behaviours, major socioeconomic costs, and reduced healthrelated quality of life in the aftermath of trauma exposure and posttraumatic stress disorders, thus defining a major challenge to any medical care system. Complex psycho-behavioural-somatic and somato-psycho-behavioural models are needed to better understand both acute and long-term effects of a perpetuating stress system on physical health.

PTSD-associated psychological and somatic comorbidities have to be considered more adequately by integrative treatment approaches. Although empirical evidence shows that psychotherapeutic (in particular, cognitive-behavioural and psychodynamic) and psychopharmacological strategies (in particular, antidepressants) may improve physical functioning in patients with medically unexplained, somatoform symptoms, bodily distress syndromes after early and current trauma exposures (Kapfhammer 2017b, Henningsen et al. 2018), considerable scepticism must be notified as regards PTSD-related physical comorbidities. Without specially tailored interventions for PTSD-altered, life style-based unhealthy behaviours and without proper medical appreciation of chronic pathophysiological states that are regulated by their own discrete dynamics, major therapeutic effects on somatic comorbidities can unlikely be awaited through usual treatments solely validated for psychopathological and psychosocial domains of PTSD. As Schnurr (2015) quite convincingly explicated: „Even though PTSD may have increased the likelihood of an individual developing a given disorder, the biological mechanisms through which this has occurred may become independent of PTSD and nonreversible through the reduction of PTSD symptoms alone" (p. 94).

\section{Acknowledgements: None.}

\section{Conflict of interest: None to declare.}

\section{References}

1. Afari N, Ahumada SM, Wright LJ et al: Psychological trauma and functional somatic syndromes: a systematic review and meta-analysis. Psychosom Med 2014; 76:2-11

2. Amare AT, Schubert KO, Klingler-Hoffmann M, et al: The genetic overlap between mood disorders and cardiometabolic diseases: a systematic review of genome wired and candidate gene studies. Transl Psychiatry 2017; 7: e1007

3. Antelman S, Caggiula A, Gershon $S$ et al: Stressorinduced oscillation: a possible model of bi-directional symptoms of PTSD. Ann N YAcad Sci 1997; 21:296-305

4. Asselmann E, Wittchen HU, Lieb R, et al: Incident mental disorders in the aftermath of traumatic events: A prospective-longitudinal community study. J Affect Disord 2018; 227:82-89

5. Atwoli L, Platt JM, Basu A, et al: Association between lifetime potentially traumatic events and chronic physical conditions in the South African Stress and Health Study: a cross-sectional study. BMC Psychiatry 2016; 16:214

6. Atwoli L, Stein DJ, Koenen KC, McLaughlin KA: Epidemiology of posttraumatic stress disorder: prevalence, correlates and consequences. Curr Opin Psychiatry 2015; 28:307-311 
7. Brewin $C R$, Cloitre $M$, Hyland $P$, et al: A review of current evidence regarding the ICD-11 proposals for diagnosing PTSD and complex PTSD. Clin Psychol Rev 2017; 58: 1-15

8. Brown RJ: Dissociation and functional neurologic disorders. In: Hallett M, Stone J, Carson A (eds) Handbook of clinical neurology, vol 193 (3rd series) Functional neurological disorders. Elsevier, Amsterdam, 2016; pp 85-94

9. Brudey C, Park J, Wiaderkiewicz J, et al: Autonomic and inflammatory consequences of posttraumatic stress disorder and the link to cardiovascular disease. Am J Physiol Regul Integr Comp Physiol 2015; 309:R315-R321

10. Burg MM, Brandt C, Buta E, Schwartz J, et al: Risk for incident hypertension associated with posttraumatic stress disorder in military veterans and the effect of posttraumatic stress disorder treatment. Psychosom Med 2017; 79:181-188

11. Cohen BE, Marmar C, Ren L, et al: Association of cardiovascular risk factors with mental health diagnoses in Iraq and Afghanistan war veterans using VA health care. JAMA 2009; 302:489-492

12. Creed F, Henningsen P, Fink $P$ (eds): Medically unexplained symptoms, somatization and bodily distress. Developing better clinical services. Cambridge University Press, Cambridge, 2011

13. Daskalakis NP, Rijal CM, King C, Huckins LM, Ressler KJ: Recent Genetics and Epigenetics Approaches to PTSD. Curr Psychiatry Rep 2018; 20:30

14. De Hert M, Detraux J, Vancampfort D: The intriguing relationship between coronary heart disease and mental disorders. Dialogues Clin Neurosci 2018; 20:31-39

15. Domschke K: Patho-genetics of posttraumatic stress disorder. Psychiatr Danub 2012; 24:267-273

16. Edmondson D: An enduring somatic threat model of posttraumatic stress disorder due to acute life-threatening medical events. Soc Personal Psychol Compass 2014; 8:118-134

17. Edmondson D, Kronish IM, Shaffer JA, et al: Posttraumatic stress disorder and risk for coronary heart disease: a meta-analytic review. Am Heart J 2013; 166:806-814

18. Edmondson D, von Känel R: Posttraumatic stress disorder and cardiovascular disease. Lancet Psychiatry 2017; 4:320-329

19. Eller-Smith OC, Nicol AL, Christianson JA: Potential Mechanisms Underlying Centralized Pain and Emerging Therapeutic Interventions. Front Cell Neurosci 2018; 12:35

20. Esler M: Mental stress and human cardiovascular disease. Neurosci Biobehav Rev 2017; 74:269-276

21. Farr OM, Ko BJ, Joung Ke, et al: Posttraumatic stress disorder, alone or additively with early life adversity, is associated with obesity and cardiometabolic risk. Nutr Metab Cardiovasc Dis 2015; 25:479-488

22. Felitti, VJ, Anda RF, Norenberg D, et al: Relationship of childhood abuse and household dysfunction to many of the leading causes of death in adults. Am J Prev Med 1998; 14:245-258

23. Fleshner M, Crane CR: Exosomes, DAMPS and miRNA: features of stress physiology and immune homeostasis. Trends Immunol 2018; 38:768-776

24. Friedman MJ, Keane TM, Resick PA (eds): Handbook of PTSD: Science and practice. New York: Guilford, New York

25. Gershuny BS, Thayer JF: Relations among psychological trauma, dissociative phenomena, and trauma-related distress: a review and integration. Clin Psychol Rev 1999, 19:631-657
26. Gradus JL: Prevalence and prognosis of stress disorders: a review of the epidemiologic literature. Clin Epidemiol 2017; 9:251-260

27. Gupta MA: Review of somatic symptoms in post-traumatic stress disorder. Int Rev Psychiatry 2013; 25:86-99

28. Hafstad GS, Thoresen S, Wentzel-Larsen T, et al: PTSD or not PTSD? Comparing the proposed ICD-11 and the DSM-5 PTSD criteria among young survivors of the 2011 Norway attacks and their parents. Psychol Med 2017; 47:1283-1291

29. Hao G, Youssef NA, Davis CL, Su S: The role of DNA methylation in the association between childhood adversity and cardiometabolic disease. Int $J$ Cardiol 2018; 255:168-174

30. Heinzelmann M, Gill J: Epigenetic mechanisms shape the biological response to trauma and risk for PTSD: a critical review. Nurs Res Pract 2013; 417010

31. Henningsen $P$, Zipfel $S$, Herzog $W$ : Management of functional somatic syndromes. Invited review. Lancet 2007; 396:946-955

32. Henningsen P, Zipfel S, Sattel H, Creed F: Management of Functional Somatic Syndromes and Bodily Distress. Psychother Psychosom 2018; 87:12-31

33. Hughes K, Bellis MA, Hardcastle KA, et al: The effect of multiple adverse childhood experiences on health: a systematic review and meta-analysis. Lancet Pub Health 2017; 2:e356-e366

34. Jacob L, Haro JM, Koyanagi A: Post-traumatic stress symptoms are associated with physical multimorbidity: Findings from the Adult Psychiatric Morbidity Survey 2007. J Affect Disord 2018; 232:385-392

35. Kapfhammer HP: Diagnostische Konzeptualisierung von Trauma- und Stressor-bezogenen Störungen im DSM 5. Nervenarzt 2014; 85:553-563

36. Kapfhammer HP: Akute und Posttraumatische Belastungsstörung. In: Möller HJ, Laux G, Kapfhammer HP (Hrsg) Psychiatrie, Psychosomatik und Psychotherapie. 5. Aufl. Springer Verlag GmbH Deutschland, Bd. 2017a; 3:19652040

37. Kapfhammer HP: Somatoforme Störungen - somatoforme Belastungsstörung und verwandte Störungen. In: Möller HJ, Laux G, Kapfhammer HP (Hrsg) Psychiatrie, Psychosomatik und Psychotherapie. 5. Aufl. Springer Verlag GmbH Deutschland, Bd. 2017b; 3:2015-2253

38. Kapfhammer HP: Dissoziative Störungen. In: Möller HJ, Laux G, Kapfhammer HP (Hrsg) Psychiatrie, Psychosomatik und Psychotherapie. 5. Aufl. Springer Verlag GmbH Deutschland, Bd. 2017; 3:2055-2104

39. Kapfhammer HP: Der Weg von der Neurologie des ausgehenden 19. Jahrhunderts über die Psychoanalyse wieder zurück zur Neurologie des 21. Jahrhunderts? Psychologische Medizin 2018; 2:12-23

40. Katon W, Sullivan M, Walker E: Medical symptoms without identified pathology: relationship to psychatric disorders, childhood and adult trauma, and personality traits. Ann Intern Med 2001; 134: 917-925

41. Keane TM, Brief DJ, Pratt EM, Miller MW: Assessment of PTSD and its comorbidities in adults. In: Friedman MJ, Keane TM, Resick PA (eds) Handbook of PTSD. Science and practice. Guilford Press, New York/London, 2007; pp 279-305

42. Kessler RC, Sonnega A, Bromet E et al: Posttraumatic stress disorder in the National Comorbidity Survey. Arch Gen Psychiatry 1995; 52:1048-1060 
43. Kibler JL: Posttraumatic stress disorder and cardiovascular disease risk. J Trauma Dissociation 2009; 10:135-150

44. Kirmayer LJ, Kienzler H, Afana AH, Pedersen D: Trauma and disasters in social and cultural context. In: Bhugra D, Morgan C (eds) Principles of social psychiatry, 2nd ed. Wiley-Blackwell, New York, 2010; pp 155-177

45. Kivimäki M, Steptoe A: A effects of stress on the development and progression of cardiovascular disease. Nat Rev Cardiol 2018; 15:215-229

46. Koenen KC, Sumner JA, Gilsanz P, et al: Post-traumatic stress disorder and cardiometabolic disease: improving causal inference to inform practice. Psychol Med 2017; 47:209-225

47. Levy BS, Sidel VW: Adverse health consequences of the Iraq War. Lancet 2013; 381:949-958

48. Li J, Zweig KC, Brackbill RM, et al: Comorbidity amplifies the effects of post-9/11 posttraumatic stress disorder trajectories on health-related quality of life. Qual Life Res 2018; 27:651-660

49. Lohr JB, Palmer BW, Eidt CA, et al: Is post-traumatic stress disorder associated with premature senescence? A review of the literature. Am J Geriatr Psychiatry 2015, 23:709-725

50. Maaranen P, Tanskanen A, Haatainen $K$ et al: The relationship between psychological and somatoform dissociation in the general population. J Nerv Ment Dis 2005; 193:690-692

51. McFarlane AC, Ellis N, Barton $C$ et al: The conundrum of medically unexplained syndromes: questions to be answered. Psychosomatics 2008; 49:369-377

52. Maercker A, Brewin CR, Bryant RA, Cloitre $M$ et al: Proposals for mental disorders specifically associated with stress in the International Classification of Diseases11. Lancet 2013; 381:1683-1685

53. McEwen BS, Gray J, Nasca C: Recognizing Resilience: Learning from the Effects of Stress on the Brain. Neurobiol Stress 2015; 1:1-11

54. McEwen BS, Stellar E: Stress and the individual: Mechanisms leading to disease. Arch Intern Med 1993; 153: 2093-2101

55. McLeay SC, Harvey WM, Romaniuk MN, et al: Physical comorbidities of post-traumatic stress disorder in Australian Vietnam War veterans. Med J Aust 2017; 206:251-257

56. Mellon SH, Gautam A, Hammamieh R, et al: Metabolism, metabolomics, and inflammation in posttraumatic stress disorder. Biol Psychiatry 2018; 83:866-875

57. Michopoulos V, Rothbaum AO, Jovanovic T, Almli LM, Bradley B, Rothbaum BO, et al: Association of CRP genetic variation and CRP level with elevated PTSD symptoms and physiological responses in a civilian population with high levels of trauma. Am J Psychiatry 2015; 172: 353-362

58. Miller MW, Lin AP, Wolf EJ, Miller DR: Oxidative stress, inflammation, and neuroprogression in chronic PTSD. Harvard Rev Psychiatry 2018; 26:57-69

59. Miller MW, Wolf EJ, Sadeh N, et al: A novel locus in the oxidative stress-related gene ALOX 12 moderates the association between PTSD and thickness of the prefrontal cortex. Psychoneuroendocrinology 2015; 62:359-36

60. Morina N, Schnyder U, Klaghofer, et al: Trauma exposure and the mediating role of posttraumatic stress on somatic symptoms in civilian war victims. BMC Psychiatry 2018, 18:92
61. Morina N, Wicherts JM, Lobbrecht J, Priebe S: Remission from post-traumatic stress disorder in adults: a systematic review and meta-analysis of long term outcome studies. Clin Psychol Rev 2014; 34:249-255

62. Morris G, Berk M, Puri BK: A comparison of neuroimaging abnormalities in multiple sclerosis, major depression and chronic fatigue syndrome (myalgic encephalomyelitis): is there a common cause? Mol Neurobiol 2017; 55:3592-3609

63. Nijenhuis ERS: Somatoform dissociation: major symptoms of dissociative disorders. J Trauma Dissociation 2000; 1:7-32

64. Norris FH, Slone LB: Epidemiology of trauma and PTSD. In: Friedman MJ, Keane TM, Resick TA (eds) Handbook of PTSD. Practice and science, 2nd ed. Guilford Press, New York, 2014; pp 100-120

65. Nusslock R, Miller GE: Early-life adversity and physical and emotional health across the lifespan: a neuro-immune network hypothesis. Biol Psychiatry 2016; 80:23-32

66. Pace TW, Heim CM: A short review on the psychoneuroimmunology of posttraumatic stress disorder: from risk factors to medical comorbidities. Brain Behav Immun 2011; 25:6-13

67. Pacella ML, Hruska B, Delahanty DL: The physical health consequences of PTSD and PTSD symptoms: a metaanalytic review. J Anxiety Disord 2013; 27:33-46

68. Passos IC, Vasconcelos-Moreno MP, Costa LG, et al: Inflammatory markers in post-traumatic stress disorder: a systematic review, meta-analysis, and meta-regression. Lancet Psychiatry 2015; 2:1002-1012

69. Pedersen SS, von Känel R, Tully PJ, Denollet J: Psychosocial perspectives in cardiovascular disease. Eur $J$ Prevent Cardiol 2018; 24:108-115

70. Penninx BWJH, Lange SMM: Metabolic syndrome in psychiatric patients: overview, mechanisms, and implications. Dialogues Clin Neurosci 2018; 20:63-72

71. Picard M, McEwen BS: Psychological stress and mitochondria: a conceptual framework. Psychosom Med 2018; 80:126-140

72. Porges $S W$ : Reciprocal influences between body and brain in the perception and expression of affect. A polyvagal perspective. In: Fosha D, Siegel DJ, Solomon M (eds) The healing power of emotion. WW Norton, New York/London, 2009; pp 27-54

73. Priebe S, Matanov A, Janković Gavrilović J, et al: Consequences of untreated posttraumatic stress disorder following war in former Yugoslavia: morbidity, subjective quality of life, and care costs. Croat Med J 2009; 50:465-475

74. Reifels L, Mills K, Dückers MLA, O'Donnell ML: Psychiatric epidemiology and disaster exposure in Australia. Epidemiol Psychiatr Sci 2017 [Epub ahead of print]

75. Rief $W$, Broadbent E: Explaining medically unexplained symptom-models and mechanism. Clin Psychol Rev 2007; 27:821-841

76. Rief W, Mills PJ, Ancoli-Israel S, Ziegler MG, Pung MA, Dimsdale JE: Overnight changes of immune parameters and catecholamines are associated with mood and stress. Psychosom Med 2010; 72:755-762

77. Roelofs K, Pasman J: Stress, childhood trauma, and cognitive functions in functional neurological disorders. In: Hallett M, Stone J, Carson A (eds) Handbook of clinical neurology, vol 193 (3rd series) Functional neurological disorders. Elsevier, Amsterdam, 2016; pp 139-155 
78. Roloefs K, Spinhoven P: Trauma and medically unexplained symptoms. Towards an integration of cognitive and neuro-biological accounts. Clin Psychol Rev 2007; 27:798-820

79. Rosellini AJ, Dussaillant F, Zubizarreta JR, Kessler RC, Rose S: Predicting posttraumatic stress disorder following a natural disaster. J Psychiatr Res 2018; 96:15-22

80. Rosenbaum S, Stubbs B, Ward PB, Steel Z, Lederman O, Vancampfort D: The prevalence and risk of metabolic syndrome and its components among people with posttraumatic stress disorder: a systematic review and meta-analysis. Metabolism 2015; 64:926-933

81. Sack M, Hopper JW, Lamprecht F: Low respiratory sinus arrhythmia and prolonged psychophysiological arousal in posttraumatic stress disorder: heart rate dynamics and individual differences in arousal regulation. Biol Psychiatry 2004; 55:284-290

82. Sack M, Lahmann C, Jaeger B, Henningsen P: Trauma prevalence and somatoform symptoms: are there specific somatoform symptoms related to traumatic experiences? $J$ Nerv Ment Dis 2007; 195:928-933

83. Salim S: Oxidative stress and the central nervous system. J Pharmacol Exp Ther 2018; 360:201-205

84. Sansone RA, Songer DA, Miller KA: Childhood abuse, mental healthcare utilization, self-harm behavior, and multiple psychiatric diagnoses among inpatients with and without a borderline diagnosis. Compr Psychiatry 2005, 46:117-120

85. Santiago PN, Ursano RJ, Gray $C L$ et al: A systematic review of PTSD prevalence and trajectories in DSM-5 defined trauma exposed populations: intentional and nonintentional traumatic events. PLoS One 2013; 8:e59236

86. Scherrer JF, Salas J, Lustman PJ, et al: The Role of Obesity in the Association Between Posttraumatic Stress Disorder and Incident Diabetes. JAMA Psychiatry 2018 [Epub ahead of print]

87. Schnurr PP, Green BL: Understanding relationships among trauma, posttraumatic stress disorder, and health outcomes. In: Schnurr PP, Green BL (eds) Trauma and health: Physical health consequences of exposure to extreme stress. American Psychological Association, Washington, DC, 2004; pp 247-275

88. Schnurr PP, Green BL, Kaltman S: Trauma exposure and physical health. In: Friedman MJ, Keane TM, Resick PA (eds) Handbook of PTSD: Science and practice. New York: Guilford, New York, 2007; pp 406-424

89. Schnurr PP, Jankowski MK: Physical health and posttraumatic stress disorder: Review and synthesis. Semin Clin Neuropsychiatry 1999; 4:295-304

90. Schnurr PP, Lunney CA, Bovin MJ, Marx BP: Posttraumatic stress disorder and quality of life: extensions of findings to veterans of war in Iraq and Afghanistan. Clin Psychol Rev 2009; 29:727-735

91. Schnurr PP, Wachen JS, Green BL, Kaltman S: Trauma exposure, PTSD, and physical health. In: Friedman MJ,
Keane TM, Resick TA (eds) Handbook of PTSD. Practice and science, 2nd ed. Guilford Press, New York, 2014; S 502-521

92. Shalev AY: Posttraumatic stress disorder and stress-related disorders. Psychiatr Clin N Am 2009; 32:687-704

93. Sheerin CM, Lind MJ, Bountress KE, et al: The genetics and epigenetics of PTSD: overview, recent advances, and future directions. Curr Opin Psychol 2017; 14:5-11

94. Siqveland J, Hussain A, Lindstrøm JC, Ruud T, Hauff E: Prevalence of posttraumatic stress disorder in persons with chronic pain: a meta-analysis. Front Psychiatry 2017; 8:164

95. Speer K, Upton D, Semple S, McKune A: Systemic lowgrade inflammation in post-traumatic stress disorder: a systematic review. J Inflamm Res 2018; 11:111-121

96. Steinert C, Hofmann M, Leichsenring F, Kruse J: The course of PTSD in naturalistic long-term studies: high variability of outcomes. A systematic review. Nord $J$ Psychiatry 2015; 69:483-496

97. Sumner JA, Duncan LE, Wolf EJ, et al: Posttraumatic stress disorder has genetic overlap with cardiometabolic traits. Psychol Med 2017; 47:2036-2039

98. Teicher MH, Samson JA, Anderson CM, Ohashi K: The effects of childhood maltreatment on brain structure, function and connectivity. Nat Rev Neurosci 2016; 17:652-666

99. Vakonaki E, Tsiminikaki $K$, Plaitis $S$, et al: Common mental disorders and association with telomere length (review). Biomed Rep 2018; 8:111-116

100. van der Hart O, Nijenhuis ERS, Steele K: The haunted self. Structural dissociation and the treatment of chronic traumatization. Norton, New York/London, 2006

101. Wirtz PH, von Känel R: Psychological stress, inflammation, and coronary heart disease. Curr Cardiol Rep 2017; 19:11

102. Wittchen HU, Gloster A, Beesd K et al: Posttraumatic stress disorder: diagnostic and epidemiological perspectives. CNS Spectr 2009; 14(suppl 1):5-12

103. Wolf EJ, Bovin MJ, Green JD, et al: Longitudinal associations between posttraumatic stress disorder and metabolic syndrome severity. Psychol Med 2016; 46:2215-2226

104. Wolf EJ, Schnurr PP: Developing comprehensive models of the effects of stress and trauma on biology, brain, behaviour, and body. Biol Psychiatry 2016a; 80:6-8

105. Wolf EJ, Schnurr PP: PTSD-related cardiovascular disease and accelerated cell aging. Psychiatr Ann $2016 b ; 46: 527-532$

106. Wulsin L, Herman J, Thayer JF: Stress, autonomic imbalance, and the prediction of metabolic risk: a model and proposal for research. Neurosci Biobehav Rev 2018; 86:12-20

107. Zass LJ, Hart SA, Seedat S, et al: Neuroinflammatory genes associated with post-traumatic stress disorder: implications for comorbidity. Psychiatr Genet 2017; 27:1-16

Correspondence:

Prof. Hans-Peter Kapfhammer, MD, PhD

Department of Psychiatry and Psychotherapeutic Medicine, Medical University of Graz

Auenbruggerplatz 31, A-8036 Graz, Austria

E-mail: Hans-peter.kapfhammer@klinikum-graz.at 